\title{
GCN2 regulates pancreatic $\beta$ cell mass by sensing intracellular amino acid levels
}

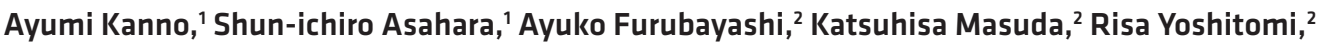 \\ Emi Suzuki, ${ }^{1}$ Tomoko Takai, ${ }^{1}$ Maki Kimura-Koyanagi, ${ }^{1}$ Tomokazu Matsuda, ${ }^{1}$ Alberto Bartolome, ${ }^{3}$ \\ Yushi Hirota, ${ }^{1}$ Norihide Yokoi, ${ }^{4}$ Yuka Inaba, ${ }^{5}$ Hiroshi Inoue, ${ }^{5}$ Michihiro Matsumoto, ${ }^{6}$ Kenichi Inoue, \\ Takaya Abe, ${ }^{7,8}$ Fan-Yan Wei, ${ }^{9}$ Kazuhito Tomizawa, ${ }^{9}$ Wataru Ogawa, ${ }^{1}$ Susumu Seino, ${ }^{4}$ \\ Masato Kasuga, ${ }^{10}$ and Yoshiaki Kido ${ }^{1,2}$ \\ 'Division of Diabetes and Endocrinology, Department of Internal Medicine, and 'Division of Metabolism and Disease, \\ Department of Biophysics, Kobe University Graduate School of Health Science, Kobe, Japan. ${ }^{3}$ Naomi Berrie Diabetes \\ Center and Department of Medicine, Columbia University, New York, New York, USA. ${ }^{4}$ Division of Molecular and Metabolic \\ Medicine, Department of Physiology and Cell Biology, Kobe University Graduate School of Medicine, Kobe, Japan. \\ ${ }^{5}$ Metabolism and Nutrition Research Unit, Institute for Frontier Science Initiative, Kanazawa University, Kanazawa, \\ Ishikawa, Japan. ${ }^{6}$ Department of Molecular Metabolic Regulation, Diabetes Research Center, Research Institute, National

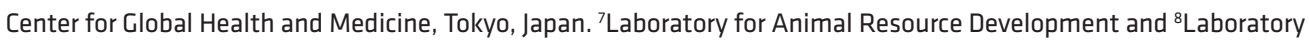 \\ for Genetic Engineering, RIKEN Center for Biosystems Dynamics Research, Kobe, Japan. ${ }^{9}$ Department of Molecular \\ Physiology, Faculty of Life Sciences, Kumamoto University, Kumamoto, Japan. ${ }^{10}$ National Center for Global Health and \\ Medicine, Tokyo, Japan.
}

EIF2AK4, which encodes the amino acid deficiency-sensing protein GCN2, has been implicated as a susceptibility gene for type 2 diabetes in the Japanese population. However, the mechanism by which GCN2 affects glucose homeostasis is unclear. Here, we show that insulin secretion is reduced in individuals harboring the risk allele of EIF2AK4 and that maintenance of GCN2-deficient mice on a high-fat diet results in a loss of pancreatic $\beta$ cell mass. Our data suggest that GCN2 senses amino acid deficiency in $\beta$ cells and limits signaling by mechanistic target of rapamycin complex 1 to prevent $\beta$ cell failure during the consumption of a high-fat diet.

Conflict of interest: The authors have declared that no conflict of interest exists.

Copyright: (ㄷ) 2020, American Society for Clinical Investigation.

Submitted: March 22, 2019

Accepted: April 1, 2020

Published: May 7, 2020.

Reference information: /CI Insight. 2020;5(9):e128820.

https://doi.org/10.1172/jci.

insight.128820.

\section{Introduction}

The increasing prevalence of type 2 diabetes mellitus (T2DM) constitutes a serious health burden worldwide. Asian individuals tend to develop diabetes at a much lower BMI than Whites (1), and impaired pancreatic $\beta$ cell function plays a greater role in T2DM in Asians than in Whites (2). A limited pancreatic $\beta$ cell capacity in Asians has also been suggested by the observation that there is little compensatory increase in $\beta$ cell mass in obese Japanese individuals, whereas there is a prominent increase in obese Whites $(3,4)$.

Analysis of single nucleotide polymorphisms (SNPs) in Japanese individuals implicated the gene for eukaryotic translation initiation factor $2 \alpha$ kinase 4 (EIF2AK4) as a candidate susceptibility gene for T2DM $(5,6)$. The protein encoded by EIF2AK4 is also known as general control nonderepressible 2 (GCN2), which — in yeast and mammals - is activated as a result of amino acid deficiency. During such a deficiency, uncharged transfer RNAs (tRNAs) bind to a regulatory region of GCN2 and induce its autophosphorylation (7, 8). Activated GCN2 phosphorylates eukaryotic initiation factor $2 \alpha$ (eIF2 $\alpha$ ) and thereby suppresses the initiation of general protein translation (9). At the same time, eIF2 $\alpha$ phosphorylation promotes the translation of specific mRNAs, such as that for activating transcription factor 4 (ATF4) (10). ATF4 activates the transcription of various genes related to amino acid metabolism, transport, and synthesis (11).

Studies have implicated GCN2 in a variety of biological processes in mammals, including those specific to the nervous system (12-14), immune system (15), and inflammation (16). Such studies have been based on the characterization of GCN2-KO $\left(\mathrm{GCN}^{-/-}\right)$mice or on mice fed an amino acid-deficient diet to activate GCN2 $(13,15,17)$. GCN2 is also thought to contribute to aspects of metabolism, having been shown to suppress lipogenesis by sensing leucine deficiency in the liver (17), to regulate hepatic gluconeogenesis in response to pyruvate administration (18), and to increase insulin sensitivity during leucine deprivation (19). 
However, the mechanism underlying the association of GCN2 with the development of T2DM is unclear.

In this study, we evaluated the relationship between the EIF2AK4 polymorphism associated with T2DM and metabolic parameters in human subjects and found that individuals with the risk allele have reduced insulin secretion. The generation and characterization of generalized and pancreatic $\beta$ cell-specific GCN2-KO ( $\left.\beta \mathrm{GCN}^{---}\right)$mice revealed that GCN2 regulates pancreatic $\beta$ cell mass in animals fed a high-fat diet (HFD). We also found that GCN2 was activated when translation of proinsulin mRNA was increased in pancreatic $\beta$ cells. Our results have, thus, uncovered a mechanism that couples amino acid sensing by GCN2 to regulation of pancreatic $\beta$ cell mass during high-fat feeding.

\section{Results}

Carriers of the risk allele for EIF2AK4 SNP rs2250402 exhibit reduced insulin secretion but no change in insulin sensitivity. A multistage genome-wide association study that began in 2002 as part of the national Millennium Genome Project in Japan revealed that EIF2AK4 SNP rs2250402 was a candidate susceptibility gene for $\operatorname{T} 2 \mathrm{DM}(5,6)$. To investigate the impact of this SNP on glucose metabolism, we conducted a 75-g oral glucose tolerance test (OGTT) and a glucose clamp test in 111 subjects who had been genotyped for rs2250402. On the basis of clinical assessment, the subjects were classified into 3 groups: normal glucose tolerance (NGT), impaired glucose tolerance (IGT), and T2DM. The background characteristics of the study participants are shown in Supplemental Table 1 (supplemental material available online with this article; https://doi.org/10.1172/jci.insight.128820DS1). The frequency of the risk allele for rs 2250402 was $38 \%$ in all subjects. In the NGT group, the carriers of the risk allele ( $A C$ and $C C$ genotypes) exhibited a significantly lower insulinogenic index, a measure of insulin secretion, during the OGTT (Figure 1A) and a significantly lower disposition index, a measure of pancreatic $\beta$ cell function in relation to insulin sensitivity $(20,21)$, during the glucose clamp test (Figure $1 C$ ) compared with those without the risk allele ( $A A$ genotype). In the IGT group, the carriers of the risk allele tended to have a lower insulinogenic index during the OGTT (Figure 1A) and showed a significantly reduced first phase of insulin secretion and disposition index during the glucose clamp test (Figure 1, B and C). Furthermore, in the NGT group, BMI was negatively correlated with the disposition index (Figure 1D). In the IGT group, this correlation was not observed (Figure 1E). The insulin sensitivity index (Supplemental Figure 1A) and glucose infusion rate (Supplemental Figure 1B) during the glucose clamp test did not differ significantly between individuals with or without the risk allele in any of the 3 groups, indicative of similar insulin sensitivity. Together, these data indicate that insulin secretion is reduced in carriers of the risk allele for rs2250402 with NGT or IGT compared with corresponding individuals without the risk allele, and that the impairment of pancreatic $\beta$ cell function in the carriers worsens with increasing BMI.

GCN2-KO mice exhibit NGT on a normal chow diet. GCN2 is highly expressed in the brain, liver, lung, and testis of mice (9). However, immunoblot analysis showed that the abundance of GCN2 was greater in mouse pancreatic islets than in the other tissues examined (Figure 2A). To examine the role of GCN2 in the development of T2DM, we generated generalized $\mathrm{GCN} 2^{-/}$mice (Supplemental Figure 2A) and investigated the impact of systemic GCN2 deficiency on glucose metabolism. GCN2 ${ }^{-/}$mice were born in a Mendelian ratio and did not display any external malformations or behavioral abnormalities (data not shown). Immunoblot analysis confirmed that the amount of GCN2 in the hypothalamus, liver, and pancreatic islets was reduced in heterozygous $\mathrm{KO}$ mice and that the protein was virtually undetectable in homozygous $\mathrm{KO}$ mice (Figure 2B). Body weight and blood glucose and serum insulin concentrations under ad libitum feeding (Supplemental Figure 3, A-C), as well as glucose and insulin responses during an OGTT (Figure 2, C and D), did not differ between $\mathrm{GCN} 2^{-/-}$and control $\left(\mathrm{GCN} 2^{+/+}\right.$) mice maintained on a normal chow diet (NCD). Furthermore, neither insulin sensitivity (Supplemental Figure 3D) nor pancreatic $\beta$ cell mass (Figure 2E) differed between the 2 genotypes. These results indicated that glucose tolerance and other metabolic parameters were not altered in GCN2 $2^{-1}$ mice fed a NCD.

GCN2-KO mice exhibit glucose intolerance and reduced pancreatic $\beta$ cell mass on a HFD. We examined the phosphorylation of GCN2 in the islets of WT mice fed a NCD or HFD. Immunoblot analysis revealed that the level of GCN2 phosphorylation in islets was greater in mice fed a HFD compared with those fed a NCD (Figure 2F), whereas GCN2 phosphorylation in the hypothalamus or liver did not differ between the 2 diets (Supplemental Figure 3E). Therefore, we examined the effects of HFD feeding on GCN2 ${ }^{-/-}$mice. Body weight, as well as blood glucose and serum insulin levels during ad libitum feeding, did not differ 
A

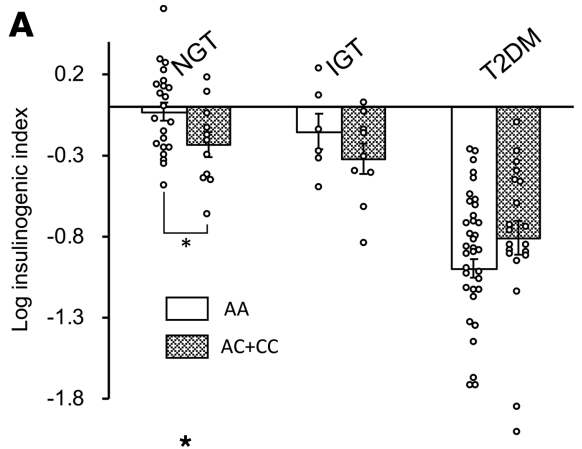

B
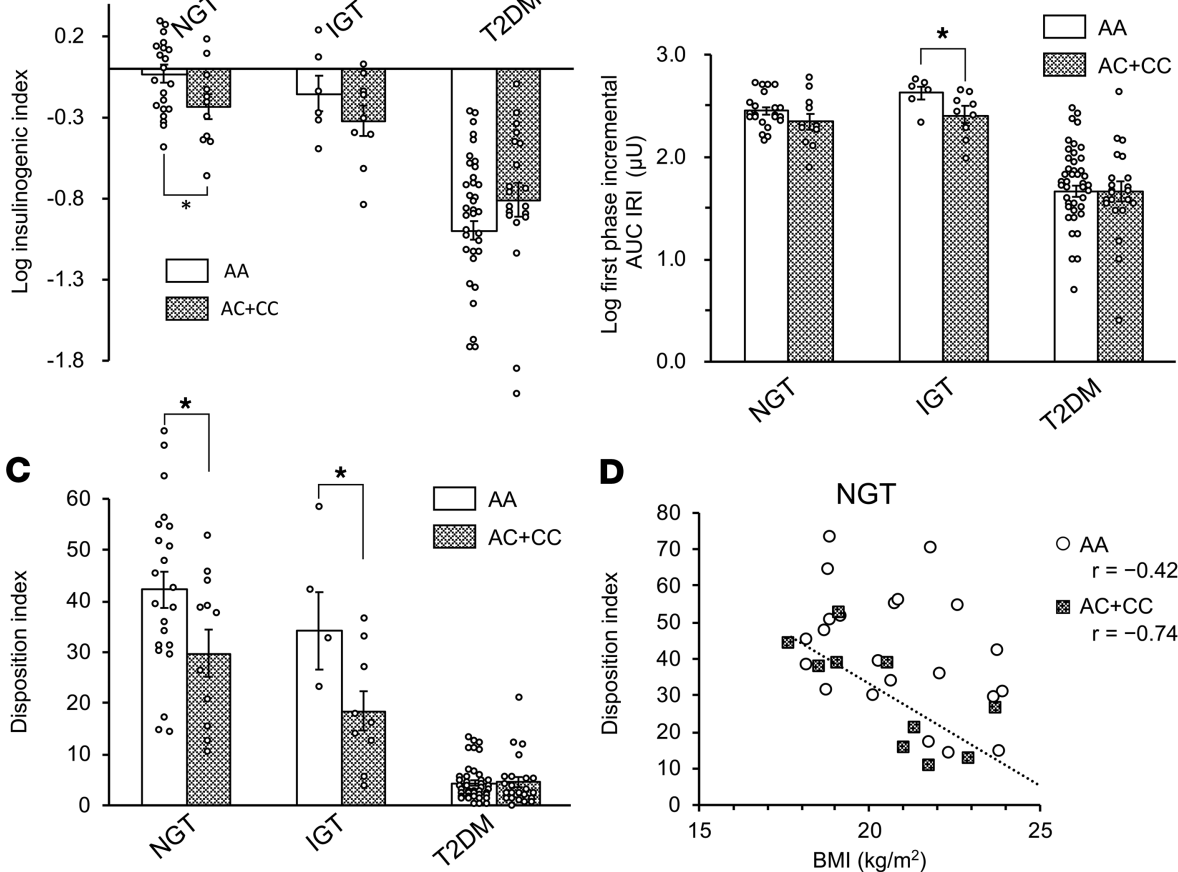

D

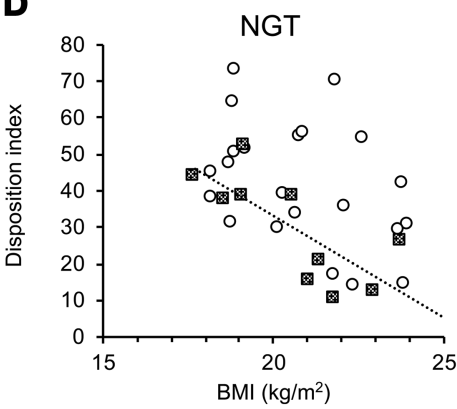

O AA $r=-0.422, P=0.663$ 중 $\mathrm{AC}+\mathrm{CC}$ $\mathrm{r}=-0.746, P=0.008$

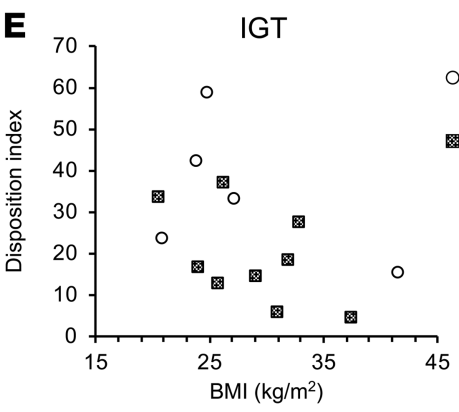

Figure 1. Carriers of the risk allele for EIF2AK4 SNP rs2250402 exhibit reduced insulin secretion but no change in insulin sensitivity. (A) Insulinogenic index determined during a $75-\mathrm{g}$ oral glucose tolerance test (OGTT) for the study participants classified according to normal glucose tolerance (NGT), impaired glucose tolerance (ICT), or type 2 diabetes mellitus (T2DM) status and whether they harbored the risk allele (C) of rs2250402. (B and C) First-phase insulin secretion and the disposition index, respectively, determined for the study subjects during a glucose clamp test. Data in $\mathbf{A}-\mathbf{C}$ are the mean \pm SEM. ${ }^{*} P<0.05$ (2-tailed Student's $t$ test). ( $\mathbf{D}$ and $\mathbf{E}$ ) Correlation between the disposition index and BMI for the study participants with NGT (D) or IGT (E). Data in D and E were analyzed by Pearson's correlation analysis. In D, the dotted line shows the regression line of AC + CC. IRI, immunoreactive insulin.

between $\mathrm{GCN}_{2}^{-/-}$and $\mathrm{GCN} 2^{+/+}$mice fed a HFD up to 24 weeks of age (Supplemental Figure 3, F-H). However, an OGTT at 24 weeks of age revealed that the blood glucose concentration was significantly higher and serum insulin concentration was significantly lower in $\mathrm{GCN} 2^{-/-}$mice compared with control mice after glucose loading (Figure 2, G and $\mathrm{H}$ ). Furthermore, immunofluorescence analysis revealed that pancreatic $\beta$ cell mass was significantly reduced in $\mathrm{GCN} 2^{-/-}$mice compared with control mice on a HFD (Figure 2I). To assess insulin resistance, we measured liver and fat weight, liver triglyceride levels, and glucose excursion during an insulin tolerance test (ITT). None of these parameters differed between $\mathrm{GCN}^{-1-}$ and control mice maintained on a HFD (Supplemental Figure 3, I-L).

To eliminate the potential effects of GCN2 loss in other tissues and to examine the specific effects of GCN2 ablation in pancreatic $\beta$ cells on glucose metabolism, we generated $\beta \mathrm{GCN}^{-/-}$mice (Supplemental Figure $2 \mathrm{~B}$ and Supplemental Figure $3 \mathrm{M}$ ). $\beta \mathrm{GCN} 2^{-/-}$mice did not exhibit any change in body weight, blood glucose concentration during ad libitum feeding (Supplemental Figure 3, N and O), or glucose metabolism (Supplemental Figure 3, P and Q) compared with control mice when maintained on a NCD. $\beta G C N 2^{-/-}$mice 
A
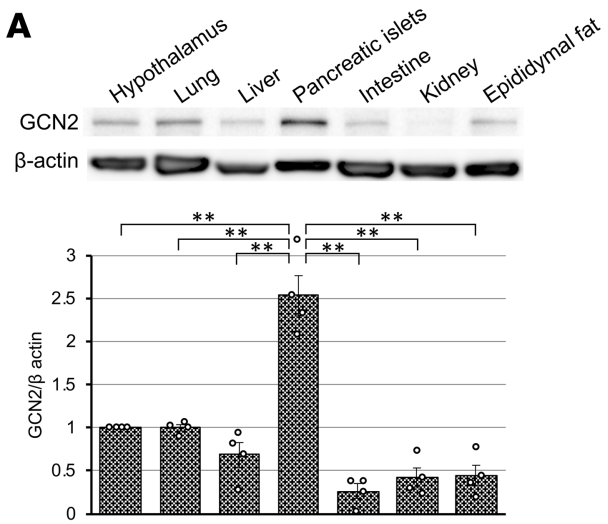

B

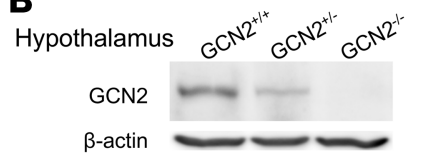

Liver

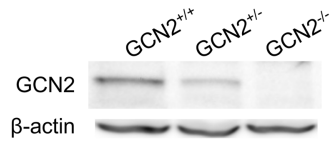

Pancreatic islets

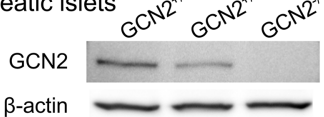

C

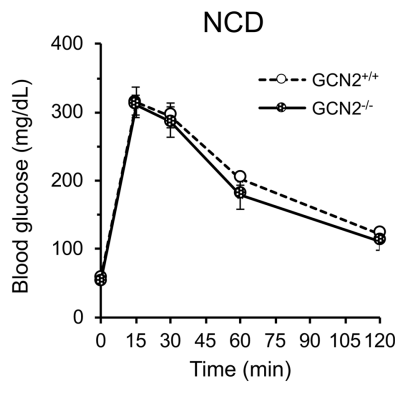

$\mathbf{F}$
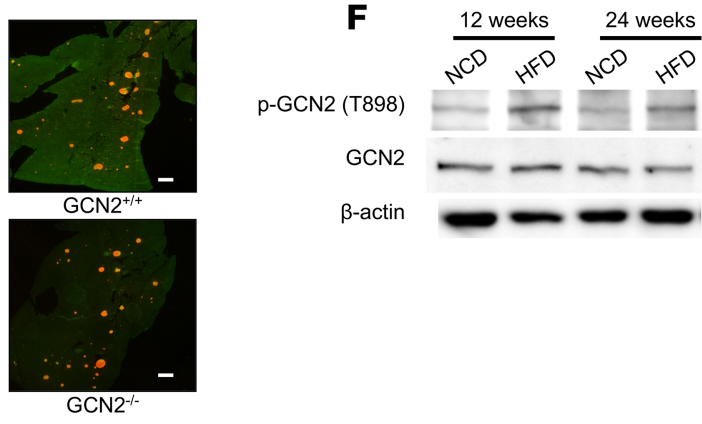

p-GCN2 (T898)

GCN2

$\beta$-actin
G
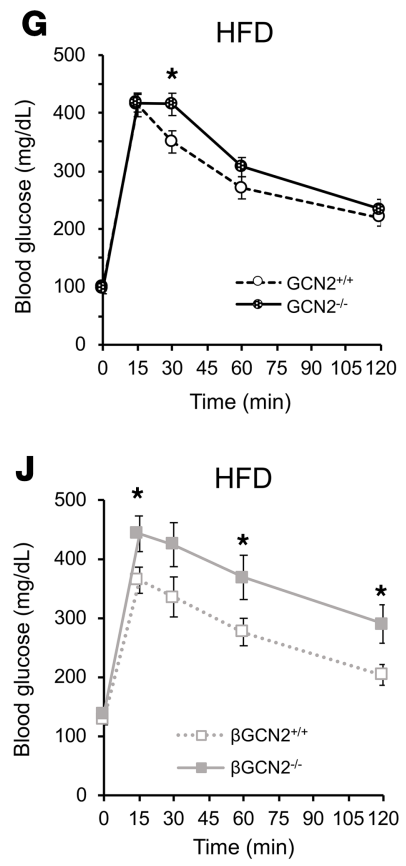
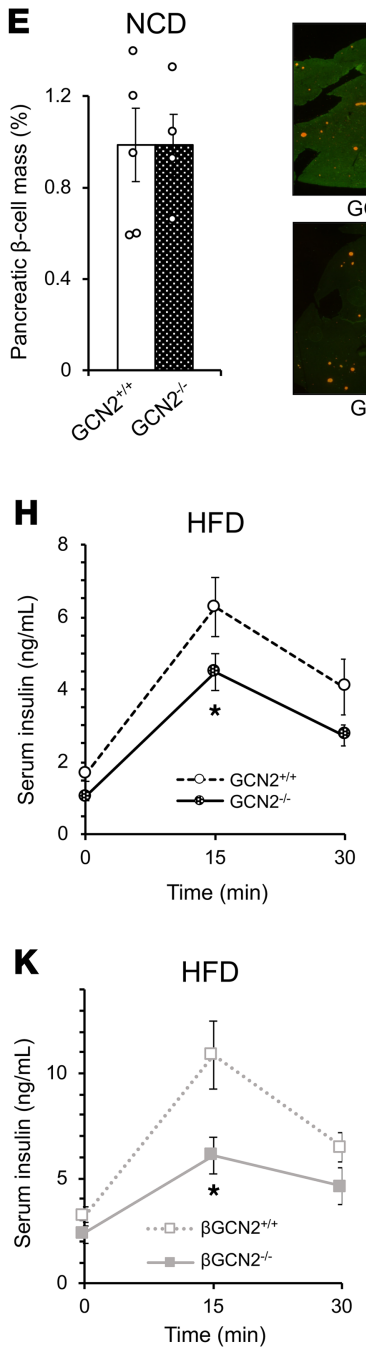
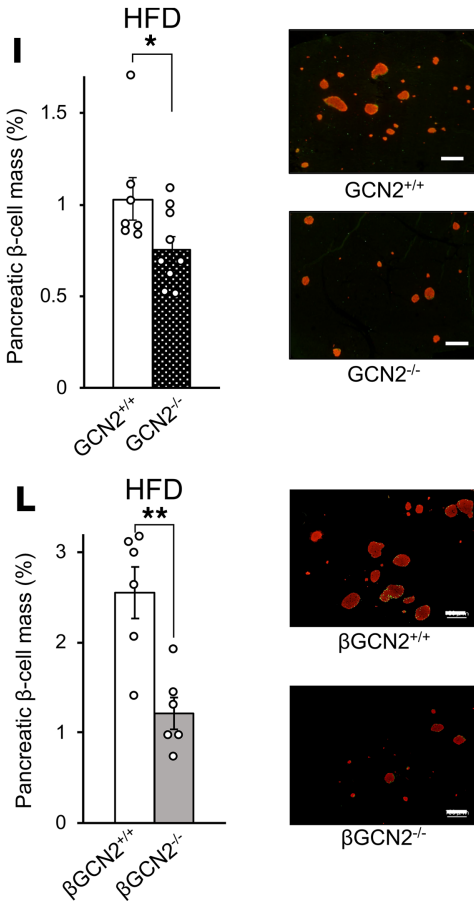

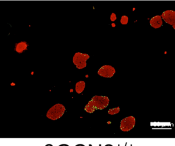

$\beta_{\mathrm{GCN}} 2^{+/+}$

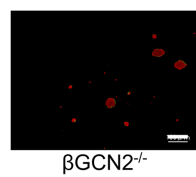

Figure 2. GCN2-KO mice exhibit glucose intolerance and reduced pancreatic $\beta$ cell mass when fed a high-fat diet (HFD). (A) Immunoblot analysis of GCN2 and $\beta$-actin in tissues of 12 -week-old WT mice (upper) and the relative GCN2/ $\beta$-actin ratio (lower). Data are the mean \pm SEM ( $n=4$ ). Quantative data for 4 independent experiments are shown. (B) Immunoblot analysis of GCN2 in tissues from 12-week-old GCN2+/+, GCN2 ${ }^{+/-}$, and GCN2-/- mice. (C-E) Blood glucose (C) and serum insulin concentrations (D) during an OCTT and immunostaining of insulin (red) and glucagon (green) in pancreatic sections (E, right) from 24-week-old normal chow diet-fed (NCD-fed) GCN2 $2^{+/+}$and GCN2 ${ }^{-/-}$mice. Quantified pancreatic $\beta$ cell mass (E, left). (F) Immunoblot analysis of phosphorylated and total GCN2 in the islets of 12- or 24-week-old WT mice fed a NCD or HFD. (G-I) Blood glucose (G), serum insulin levels (H) during an OGTT, and immunostaining of insulin (red) and glucagon (green) in pancreatic sections (I, right) from 24-week-old HFD-fed GCN2+/+ and GCN2 ${ }^{-/-}$mice. Quantified pancreatic $\beta$ cell mass (I, left). (J-L) Blood glucose (J), serum insulin levels (K) during an OGTT and immunostaining of insulin (red) and glucagon (green) in pancreatic sections (L, right) from $24-$ week-old HFD-fed $\beta$ GCN2 ${ }^{+/+}$and $\beta$ GCN2 ${ }^{-/-}$ mice. Quantified pancreatic $\beta$ cell mass ( $\mathbf{L}$, left). Scale bars: $100 \mu \mathrm{m}(\mathbf{E}, \mathbf{I}$, and $\mathbf{L})$. Quantitative data in $\mathbf{C}-\mathbf{E}$ and $\mathbf{G}-\mathbf{L}$ are the mean \pm SEM ( $n=6-11)$. ${ }^{*} P<0.05,{ }^{*} P<0.01$ (2-tailed Student's $t$ test). 
fed a HFD also did not differ from control mice with regard to body weight, blood glucose concentration during ad libitum feeding, or blood glucose lowering during an ITT (Supplemental Figure 3, R-T); however, they showed a greater increase in blood glucose and a smaller increase in serum insulin after glucose loading (Figure 2, J and $\mathrm{K}$ ), as well as reduced pancreatic $\beta$ cell mass (Figure 2L). Collectively, these data show that systemic or pancreatic $\beta$ cell-specific GCN2 ablation did not induce insulin resistance but that both types of mutant mice developed pancreatic $\beta$ cell dysfunction during maintenance on a HFD. They thus indicated that GCN2 plays an important role in maintaining glucose homeostasis during HFD feeding.

Mechanistic target of rapamycin complex 1 signaling is enhanced in the islets of GCN2-1- mice fed a HFD. To gain an insight into the molecular mechanism underlying the reduction in pancreatic $\beta$ cell mass in GCN2 ${ }^{-/-}$ mice fed a HFD, we examined intracellular signaling in isolated islets. The phosphorylation of mechanistic target of rapamycin (mTOR, on $\mathrm{Ser}^{2448}$ ) and its downstream signaling targets ribosomal protein S6 (on $\mathrm{Ser}^{235 / 236}$ ) and eIF4E binding protein 1 (4EBP1, on $\mathrm{Thr}^{37 / 46}$ ) was markedly increased (Figure 3A), whereas that of upstream molecules of the insulin signaling pathway, including Akt (on $\mathrm{Thr}^{308}$ and $\mathrm{Ser}^{473}$ ) and glycogen synthase kinase $3 \beta\left(\mathrm{GSK} 3 \beta\right.$, on $\mathrm{Ser}^{9}$ ), was decreased (Figure $3 \mathrm{~B}$ ) in the islets of GCN2 ${ }^{-/-}$mice fed a HFD compared with control mice. These findings suggested that signaling by mTOR complex 1 (mTORC1) was enhanced, whereas proximal insulin signaling was attenuated in the islets of HFD-fed $\mathrm{GCN}^{-/-}$mice. The size of individual pancreatic $\beta$ cells was significantly increased by $\sim 50 \%$, and $\beta$ cell number was reduced by $\sim 70 \%$ in $\mathrm{GCN}^{-/-}$mice fed a HFD compared with control mice (Figure 3, $\mathrm{C}$ and D). The number of $\beta$ cells positive for the proliferation marker Ki67 was decreased (Figure 3E), while the number of apoptotic $\beta$ cells detected by the TUNEL assay was increased (Figure 3F) in the islets of HFDfed GCN2 $2^{--}$mice. The extent of mTORC1 signaling was not significantly altered in the liver or hypothalamus of HFD-fed GCN2 $2^{-/-}$mice compared with control mice (Supplemental Figure 4), with both of these tissues having been shown to express GCN2 in WT mice (Figure 2A).

We investigated intracellular signaling in INS-1 rat pancreatic $\beta$ cells depleted of GCN2 by transfection with a specific siRNA (Figure 3, G and $\mathrm{H}$ ). The extent of mTORC1 signaling was also increased by GCN2 knockdown in these cells, whereas that of upstream insulin signaling was attenuated (Figure 3, I and J). Our previous analysis of mice in which mTORC1 signaling is chronically activated in pancreatic $\beta$ cells, as a result of the specific $\mathrm{KO}$ of tuberous sclerosis complex 2 (TSC2) in these cells, revealed the presence of a negative feedback loop in which mTORC1 signaling induces the degradation of insulin receptor substrate 2 (IRS2) and reduction of pancreatic $\beta$ cell mass $(22,23)$. We also observed decreased IRS2 levels following GCN2 knockdown in INS-1 cells (Figure 3J). Our present results, therefore, suggest the possibility that the enhancement of mTORC1 signaling may play an important role in the attenuation of insulin signaling in the islets of HFD-fed GCN2 $2^{-/}$mice.

Activation of mTORC1 signaling is due to impaired ATF4 and Sestrin2 expression in the islets of GCN2-1- mice fed a HFD. We investigated the molecular mechanism responsible for the enhanced mTORC1 signaling in the islets of HFD-fed GCN2 ${ }^{-/}$mice. Activated GCN2 phosphorylates eIF2 $\alpha$ (24) and thereby elicits a selective increase in ATF4 mRNA translation (25). Indeed, we found that ATF4 expression was attenuated in the islets of HFD-fed GCN2-/- mice and in GCN2-knockdown INS-1 cells (Figure 4, A and B). Therefore, we examined the effects of reduced ATF4 expression in INS-1 cells. ATF4 knockdown resulted in the upregulation of mTORC1 signaling and downregulation of insulin signaling in INS-1 cells (Figure $4 \mathrm{C}$ ), indicating that the increase in mTORC1 signaling induced by the loss of GCN2 is due to a reduction of ATF4 expression. ATF4 promotes the expression of a variety of genes related to amino acid synthesis and transport, as well as to the synthesis of aminoacyl tRNAs (11). We hypothesized that the loss of the expression of one of these target genes was responsible for the increase in mTORC1 signaling induced by a deficiency of GCN2, with the results of previous studies suggesting the genes regulated in development and DNA damage response 1 (REDD1), growth arrest and DNA damage-inducible protein 34 (GADD34), and Sestrin 2 as possible candidates (26-28). We found that Sestrin2 expression was significantly reduced in the islets of HFD-fed GCN2 ${ }^{-/-}$mice, as well as in GCN2-knockdown or ATF4-knockdown INS-1 cells (Figure 4, D-F). Sestrin2 binds to the GATOR2 protein complex, which is an inhibitor of GATOR1; GATOR1 binds to and inhibits RagB GTPases, ultimately leading to mTORC1 inhibition (28-31). We found that mTORC1 signaling was increased and insulin signaling decreased in Sestrin2-knockdown INS-1 cells (Figure 4G). In addition, the forced expression of Sestrin2 rescued the changes in mTORC1 and insulin signaling induced by GCN2 knockdown in INS-1 cells (Figure 4H). These findings indicated that, in the islets of HFD-fed GCN2 ${ }^{-/-}$mice and in GCN2-knockdown INS-1 cells, mTORC1 signaling is enhanced as a result of attenuated inhibition by Sestrin2, which is attributable to reduced ATF4 mRNA translation. 
A
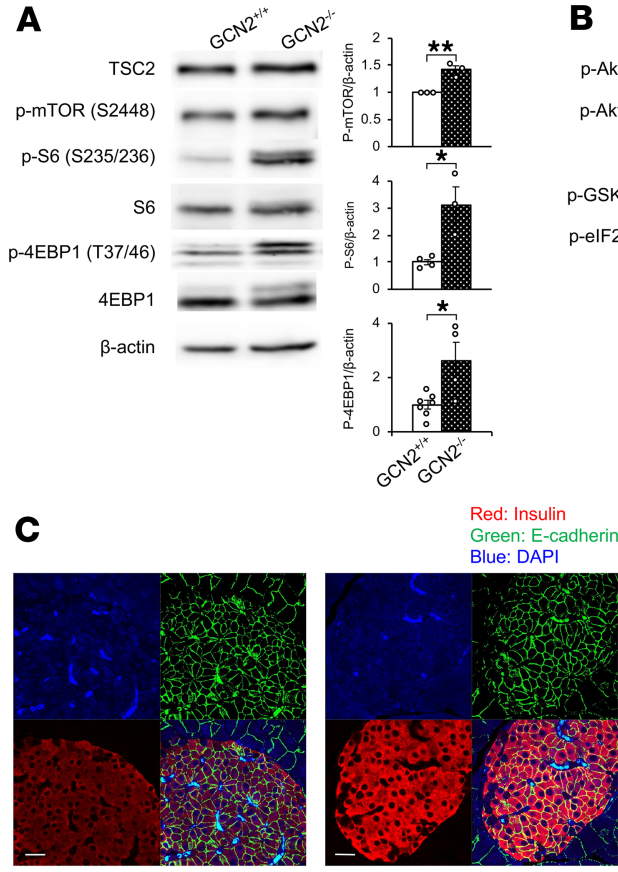

$\mathrm{GCN}^{+/+}$
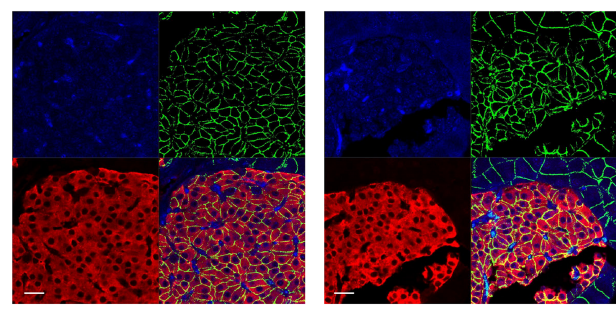

GCN2-/-

G
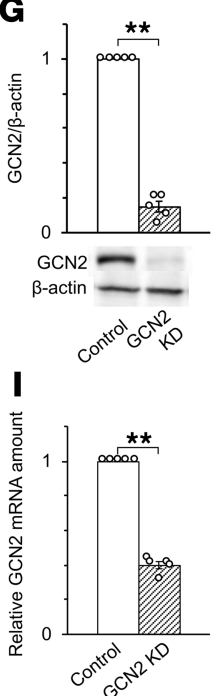
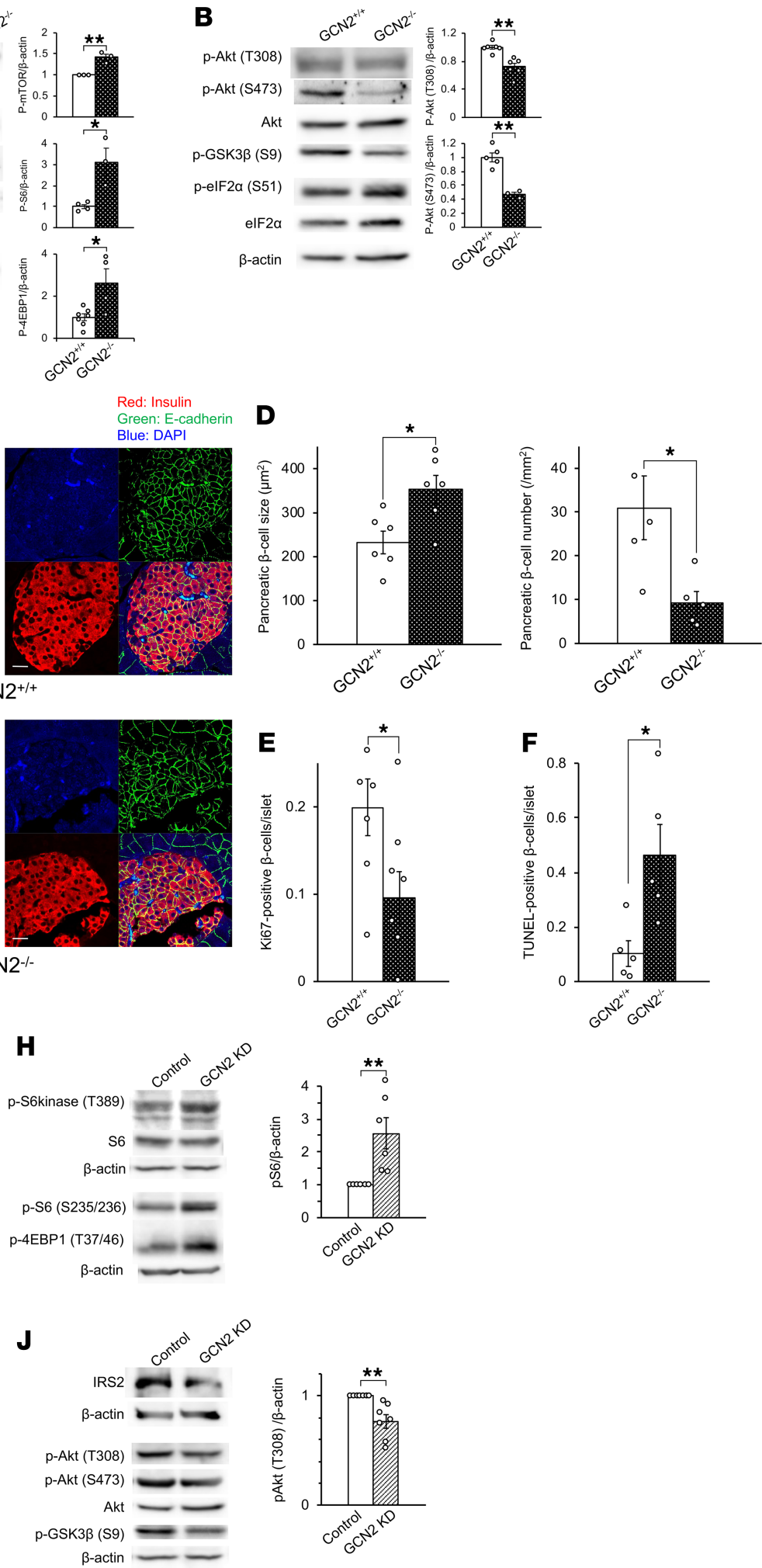

Figure 3. mTORC1 signaling is enhanced in the islets of GCN2 ${ }^{-1-}$ mice fed a HFD. (A and B) Immunoblot analysis of mTORC1 (A) and insulin (B) signaling in pancreatic islets of 24-week-old HFD-fed GCN2+/+ or $\mathrm{CCN2} 2^{-1-}$ mice. Duplicate samples in $\mathbf{A}$ and $\mathbf{B}$ were run on the same blot; the samples in $\mathbf{A}$ share the same loading control as in B. Representative blots and quantitative data for 3-5 independent experiments are shown. (C) Immunostaining of insulin and $\mathrm{E}$-cadherin in pancreatic sections from mice as in A. Scale bars: 20 $\mu \mathrm{m}$. (D) Size of individual $\beta$ cells was determined from the insulin-positive area divided by the number of nuclei in insulin-positive cells ( $n=6$ each). The number of $\beta$ cells was determined from the number of $\beta$ cells divided by the total pancreatic area ( $n$ $=5$ each). (E) The number of Ki67 $\beta$ cells per islet in 16-week-old HFD-fed $\mathrm{GCN2}^{+/+}(n=7)$ and $\mathrm{CCN2}^{-/-}(n=8)$ mice. (D) The number of apoptotic $\beta$ cells per islet in mice as in $\mathbf{D}(n=$ 5 each) was determined with the TUNEL assay. ( $\mathbf{G}$ and $\mathbf{H}$ ) Immunoblot analysis (G) and RT-PCR analysis (H) ( $n=5$ each) of GCN2 in INS- 1 cells transfected with a scramble siRNA (control) or GCN2 siRNA (GCN2 KD). (I and J) Immunoblot analysis of mTORC1 (I) and insulin (J) signaling in cells as in $\mathbf{G}$ following 24 -hour deprivation of leucine, lysine, and arginine. Representative blots and quantitative data from 3-5 independent experiments are shown. All quantitative data are the mean \pm SEM. ${ }^{*} P<0.05,{ }^{* *} P<0.01$ ( 2 -tailed Student's $t$ test)

Phosphorylation of GCN2 is increased in pancreatic $\beta$ cells when proinsulin $m R N A$ translation is enhanced. We examined the mechanism underlying the increased phosphorylation of GCN2 in the islets of WT mice fed a HFD. We first found that glucose, but not other insulin secretagogues, increased GCN2 phosphorylation in INS-1 cells (Figure 5A). Previous studies have shown that glucose increases proinsulin mRNA translation in pancreatic $\beta$ cells $(32,33)$, which can be inhibited by cycloheximide (34). We found that glucose-induced GCN2 phosphorylation was also suppressed by the translation inhibitors cycloheximide and 
A

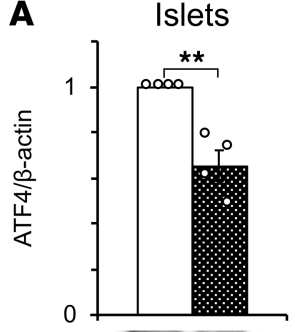

ATF4 $\rightleftharpoons$

$\beta$-actin -

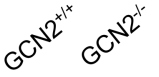

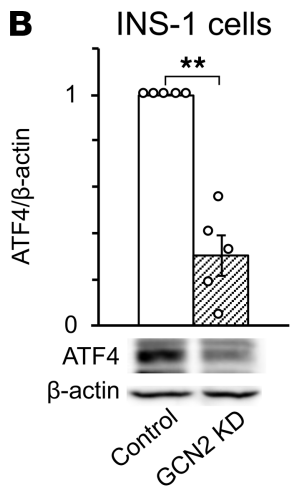

E
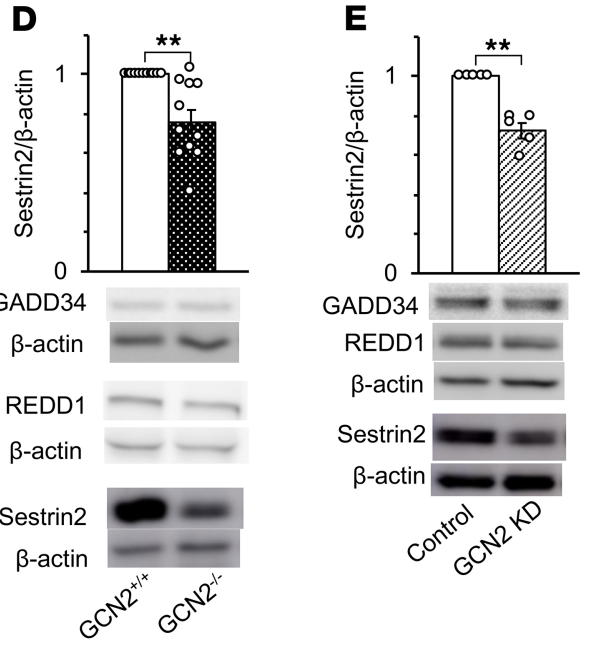

C

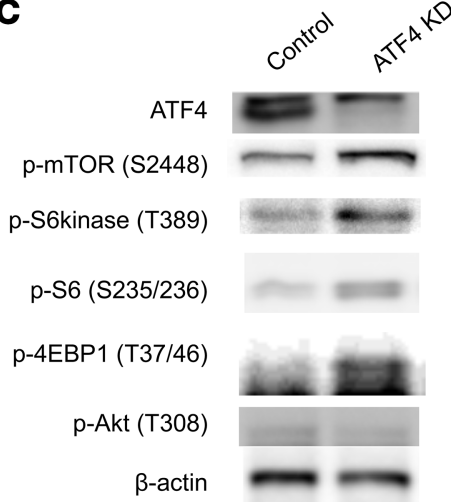

$\mathbf{F}$

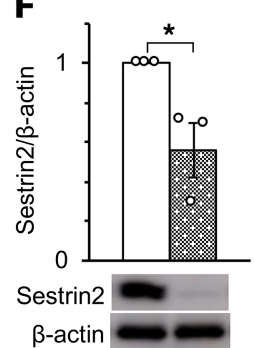

G
Sestrin2

p-mTOR (S2448)

p-S6 (S235/236)

$\beta$-actin

p-S6kinase (T389)

p-4EBP1 (T37/46)

IRS2

p-Akt (T308)

$\beta$-actin
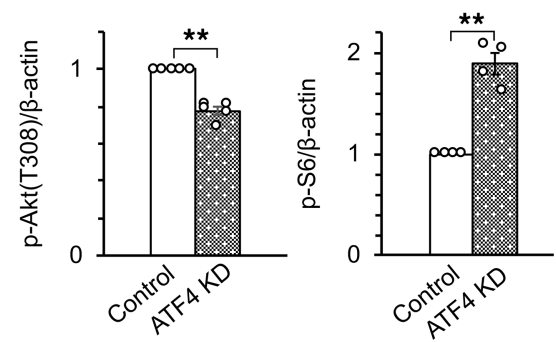

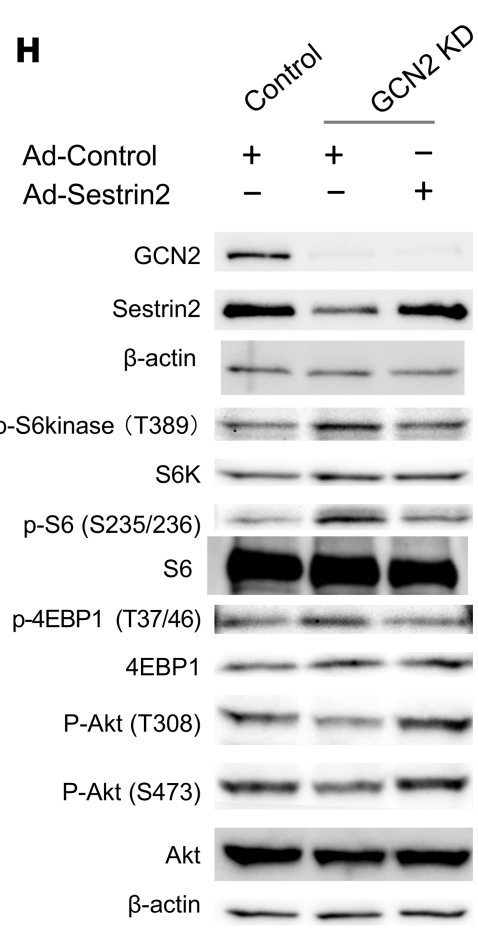

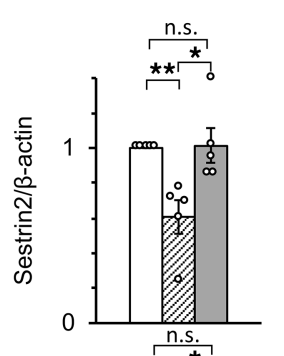

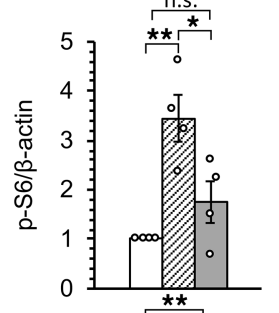

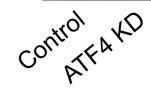

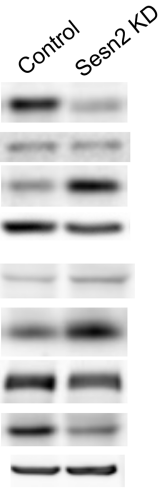

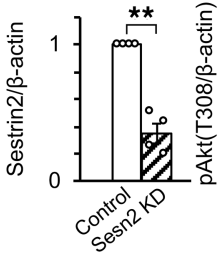
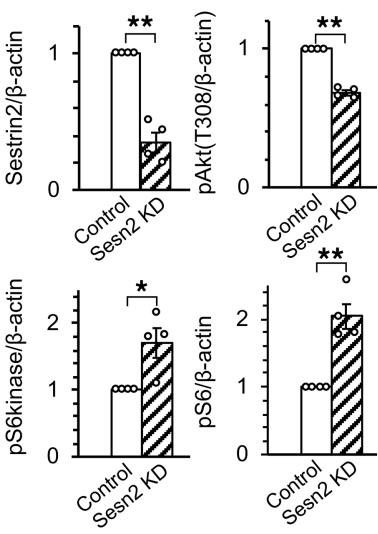

Figure 4. Activation of mTORC1 signaling is the result of impaired ATF4 and Sestrin2 expression in HFD-fed GCN2 ${ }^{-/-}$mice. (A and B) Immunoblot analysis of ATF4 in pancreatic islets of 24-week-old HFD-fed GCN2 ${ }^{+/+}$and $\mathrm{CCN2}^{-1-}$ mice (A) and in INS-1 cells transfected with scramble (control) or GCN2 siRNAs (GCN2 KD) (B). Duplicate samples in B and Figure 3j were run on same blot; the samples in $\mathbf{B}$ share the same loading control as in Figure 3J. (C) Immunoblot analysis of mTORC1 and insulin signaling in INS-1 cells transfected with scramble or ATF4 siRNAs. (D and E) Pancreatic islets of mice as in $\mathbf{A}(\mathbf{D})$ and INS-1 cells transfected with scramble or GCN2 siRNAs (E) were subjected to immunoblot analysis with antibodies to GADD34, REDD1, and Sestrin2. (F) INS-1 cells transfected with scramble or ATF4 siRNAs were subjected to immunoblot analysis with antibodies to Sestrin2. (G) Immunoblot analysis of mTORC1 and insulin signaling in INS-1 cells transfected with scramble or Sestrin2 (Sesn2) siRNAs. (H) Immunoblot analysis of mTORC1 and insulin signaling in INS-1 cells transfected with scramble or GCN2 siRNAs, infected with an adenovirus (Ad) encoding Sestrin2 or a control virus. In B, C, E, F, G, and H, INS-1 cells were deprived of leucine, lysine, and arginine for 24 hours. In all panels, representative blots and quantitative data (mean \pm SEM from at least 4 independent experiments) normalized by the amount of $\beta$-actin are shown. ${ }^{*} P<0.05,{ }^{* *} P<$ 0.01 (2-tailed Student's $t$ test). 
puromycin (Figure 5B and Supplemental Figure 5A). Among the insulin secretagogues tested, only glucose increased proinsulin biosynthesis in INS-1 cells (Figure 5C). Refeeding WT mice deprived of food overnight with normal chow increased GCN2 phosphorylation (Figure 5D) and proinsulin biosynthesis (Supplemental Figure 5B) in pancreatic islets. Moreover, GCN2 phosphorylation (Figure 2F) and proinsulin mRNA translation (34) were enhanced in the islets of WT mice fed a HFD. Given that GCN2 phosphorylation appears to be associated with proinsulin mRNA translation in vivo and in vitro, we hypothesized that GCN2 becomes phosphorylated when proinsulin biosynthesis is enhanced in pancreatic $\beta$ cells.

Amino acid levels are reduced and uncharged $t R N A$ levels are increased in the islets of mice fed a HFD. To explore further the mechanism responsible for GCN2 phosphorylation in the islets of HFD-fed mice, we applied mass spectrometry (MS) to measure amino acid levels in WT mice fed a NCD or HFD, given that GCN2 is phosphorylated in response to the binding of uncharged tRNA. The concentrations of several amino acids were significantly increased or decreased in the serum of mice fed a HFD compared with those fed a NCD (Supplemental Figure 6A). The decrease in amino acid concentrations appeared to reflect differences in amino acid composition between the 2 diets (Supplemental Table 2). Amino acid concentrations in the hypothalamus did not differ significantly between mice fed either diet (Supplemental Figure 6B). In the liver, the concentrations of a majority of amino acids were increased by the HFD (Supplemental Figure $6 \mathrm{C})$. On the other hand, in pancreatic islets, the concentrations of most amino acids tended to be or were significantly decreased in mice fed a HFD (Figure 6A). We examined whether the reduced concentrations of amino acids in the islets of HFD-fed mice might result from a change in the expression of amino acid transporters. However, with the exception of Slc1a5 mRNA, there was no difference in the abundance of mRNAs for any of the transporters examined between the islets of mice fed either diet (Supplemental Figure 6D). Finally, with the use of MS or RNA blot analysis, we found that the levels of most charged tRNAs were decreased in the islets of mice fed a HFD compared with those fed a NCD (Figure 6B and Supplemental Figure $6 \mathrm{E}$ ). In summary, amino acid concentrations were reduced and uncharged tRNA levels were increased in the islets of mice fed a HFD.

\section{Discussion}

In this study, we have shown that an SNP of EIF2AK4 that is associated with T2DM in the Japanese population is associated with reduced insulin secretion. The protein encoded by EIF2AK4, GCN2, was found to be abundant in mouse pancreatic islets, and generalized as well as pancreatic $\beta G C N 2^{-/-}$mice exhibited glucose intolerance, impaired insulin secretion, and reduced pancreatic $\beta$ cell mass when maintained on a HFD. We found that mTORC1 signaling was upregulated and insulin signaling was downregulated in the islets of $\mathrm{GCN}^{-/-}$mice and that the reduced expression of ATF4 and Sestrin2 was likely responsible for this dysregulation of mTORC1 signaling. Consumption of a HFD resulted in increased proinsulin mRNA translation in pancreatic $\beta$ cells of WT mice, which likely increased GCN2 phosphorylation through the depletion of amino acids and increase in the proportion of uncharged tRNAs. The activation of GCN2 then led to the consecutive upregulation of ATF4 and Sestrin2 expression and suppression of mTORC1 signaling. However, pancreatic $\beta$ cells of GCN2-KO mice were not able to sense such HFD-induced amino acid depletion and the accumulation of uncharged tRNAs, and so ATF4 and Sestrin2 expression was not upregulated and mTORC1 signaling was enhanced. We propose that such chronically enhanced mTORC1 signaling results in a reduction of pancreatic $\beta$ cell mass through the negative regulation of insulin signaling (Figure 7).

We have previously shown that insulin signaling in pancreatic $\beta$ cells is essential for the regulation of pancreatic $\beta$ cell mass $(22,35)$. On the other hand, chronic enhancement of mTORC1 signaling results in a reduction of pancreatic $\beta$ cell mass $(22,23)$. However, suppression of mTORC1 signaling by genetic modifications or rapamycin administration has been reported to decrease pancreatic $\beta$ cell mass (36-38). This contradiction may be due to the extent or duration of the suppression of mTORC1 signaling. It has also been reported that rapamycin administration could be beneficial or toxic, depending on its duration (39). We consider that "appropriate" regulation of mTORC1 signaling is desirable for pancreatic $\beta$ cells, and chronic activation or suppression of mTORC1 signaling adversely affects cellular survival and proliferation.

Increased mTORC1 signaling also increases pancreatic $\beta$ cell size $(22,40)$, and a decreased level of insulin signaling reduces the number of pancreatic $\beta$ cells $(22,35,41)$. These $\beta$ cell phenotypes were also observed in our GCN2 $2^{-/-}$mice maintained on a HFD. 


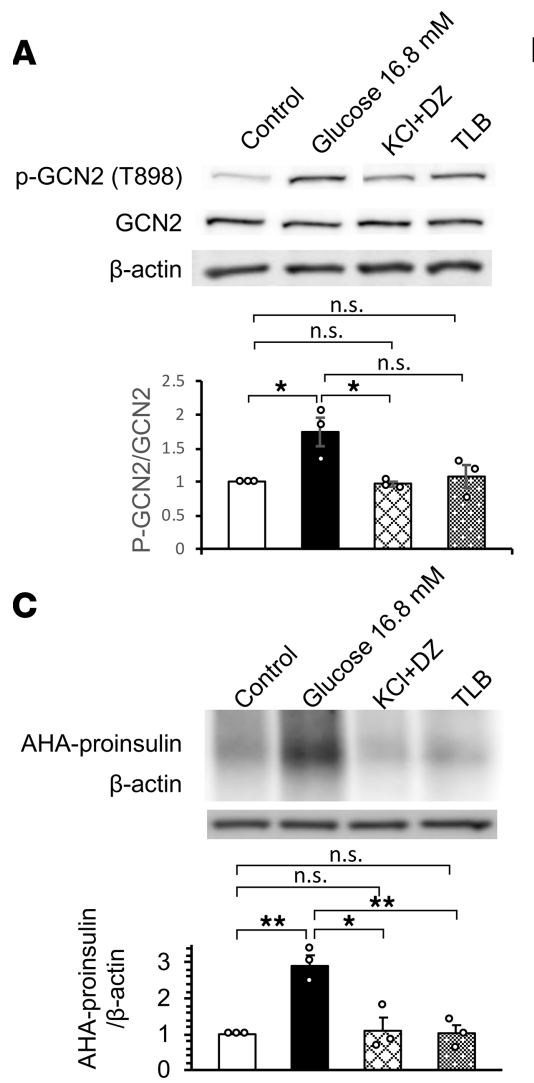

B
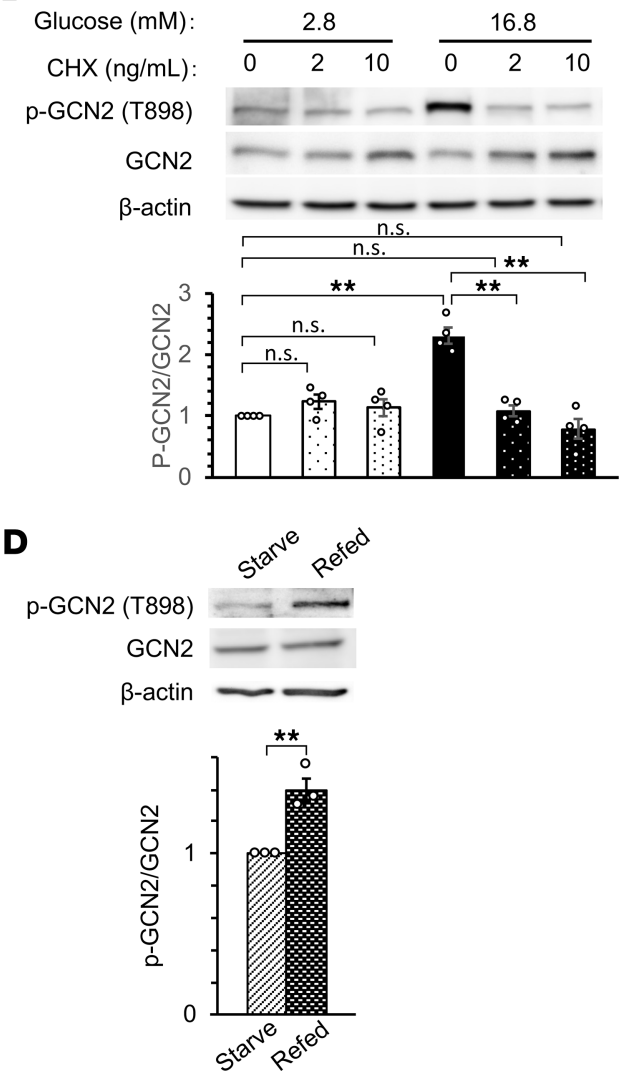

Figure 5. GCN2 phosphorylation is increased in pancreatic $\beta$ cells when proinsulin mRNA translation is enhanced. (A) Immunoblot analysis of GCN2 phosphorylation in INS-1 cells that had been maintained in the presence of 2.8 $\mathrm{mM}$ glucose and then stimulated with $16.8 \mathrm{mM}$ glucose, with $30 \mathrm{mM} \mathrm{KCl}$ plus $250 \mu \mathrm{M}$ diazoxide (DZ), or with $500 \mu \mathrm{M}$ tolbutamide (TLB) for 1 hour. (B) Immunoblot analysis of GCN2 phosphorylation in INS-1 cells that had been maintained in the presence of $2.8 \mathrm{mM}$ glucose and then incubated for 1 hour in the presence of 2.8 or $16.8 \mathrm{mM}$ glucose and the indicated concentrations of cycloheximide (CHX). (C) Cells treated as in A were analyzed for nascent translation of proinsulin mRNA by L-azidohomoalanine (AHA) labeling and immunoprecipitation with antibodies to insulin. (D) Immunoblot analysis of GCN2 phosphorylation in pancreatic islets isolated from 16-week-old WT mice that had been deprived of food overnight for 16 hours and then refed (or not) with normal chow for 2 hours. In all panels, representative blots and relative quantitative data (mean \pm SEM) for at least 3 independent experiments are shown. ${ }^{*} P<0.05$, ${ }^{* *} P<0.01$ (2-tailed Student's $t$ test).

Various upstream inputs to mTORC1 signaling, including growth factors, energy levels, and amino acids, have been identified. The activity of mTORC1 is regulated by 2 types of small GTPases, namely, Rheb, which is regulated by the TSC complex, and members of the Rag family $(42,43)$. Amino acids activate mTORC1 via Rag proteins $(44,45)$. Sestrin2 is a negative regulator of mTORC1 signaling that changes the localization of the mTORC1 complex via GATOR, which suppresses the activity of Rag GTPases (28-30). GCN2 and mTORC1 both function as amino acid-sensing kinases, and our results now suggest that a sensor of amino acid deficiency, GCN2, controls mTORC1 signaling via Rag, which is necessary for amino acids to activate mTORC1 signaling. The activity of mTORC1 is increased in the islets of HFD-fed mice in a manner dependent on the TSC2/Rheb/mTORC1 pathway $(22,46,47)$, and we have now shown that a lack of GCN2 further increases mTORC1 signaling via the Sestrin $2 / \mathrm{Rag} / \mathrm{mTORC} 1$ pathway. The activity of mTORC1 has recently been shown to be increased in the islets of patients with T2DM (48). Thus, our results have revealed a previously unrecognized role for GCN2 in limiting the activation of mTORC1 signaling in pancreatic $\beta$ cells, thereby preventing pancreatic $\beta$ cell failure during high-fat feeding. Some reports have shown that GCN2 has roles in the regulation of cell size and mass $(16,49)$ independently of mTORC 1 signaling, so we suspect that pathways other than those mediated by mTORC1 signaling might affect the regulation of pancreatic $\beta$ cell mass in $\mathrm{GCN}^{-/-}$mice. 
A
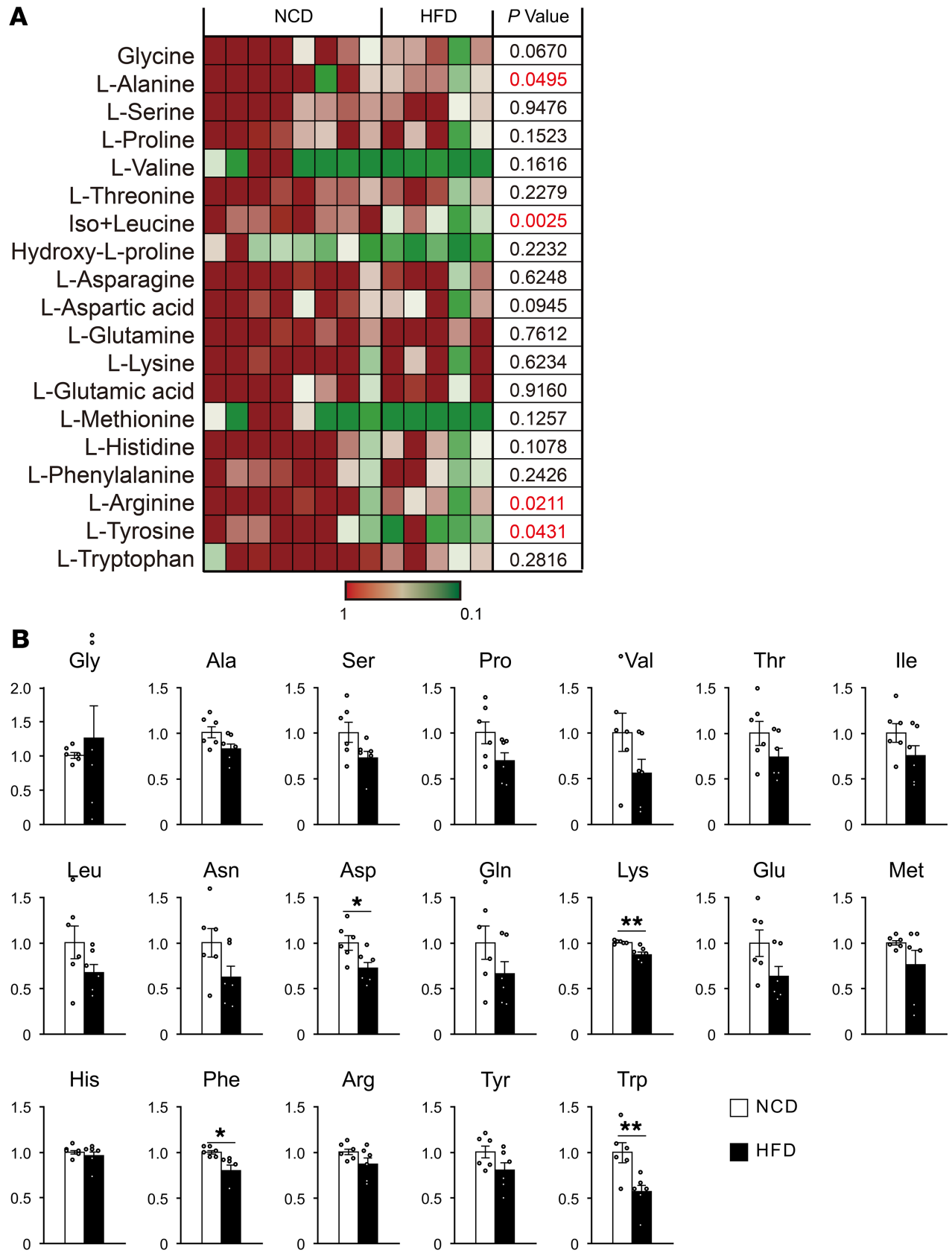

Figure 6. Amino acid levels are reduced and uncharged tRNA levels are increased in the islets of mice fed a HFD. (A) Concentrations of amino acids in pancreatic islets (NCD, $n=8$; HFD, $n=5$ ) of 24-week-old NCD- or HFD-fed WT mice were determined by metabolome analysis. Relative values are presented according to the indicated color scale for individual animals, with $P$ values less than 0.05 shown in red. (B) Relative amounts of amino acids liberated from tRNA isolated from the pancreatic islets of 24-week-old NCD- or HFD-fed WT mice were determined by metabolome analysis ( $n=6,1$ sample contains islets from 2 mice). Data in $\mathbf{B}$ are the mean \pm SEM. ${ }^{*} P<0.05,{ }^{*} P<$ 0.01 (Student's $t$ test). Gly, glycine; Ala, alanine; Ser, serine; Pro, proline; Val, valine; Thr, threonine; Ile, isoleucine; Leu, leucine; Asn, asparagine; Asp, aspartic acid; Gln, glutamine; Lys, lysine; Glu, glutamic acid; Met, methionine; His, histidine; Phe, phenylalanine; Arg, arginine; Tyr, tyrosine; Trp, tryptophan.

Our GCN2 $2^{-/-}$mice did not show any pathognomonic phenotypes when fed a NCD, but their pancreatic $\beta$ cell mass was reduced when fed a HFD. Our findings indicative of a correlation between proinsulin mRNA translation and GCN2 phosphorylation levels in vivo and in vitro suggest that GCN2 might undergo phosphorylation in pancreatic $\beta$ cells in response to increased proinsulin biosynthesis. The binding of uncharged tRNAs is the only known mechanism for the activation of GCN2 $(25,50,51)$, and we detected 


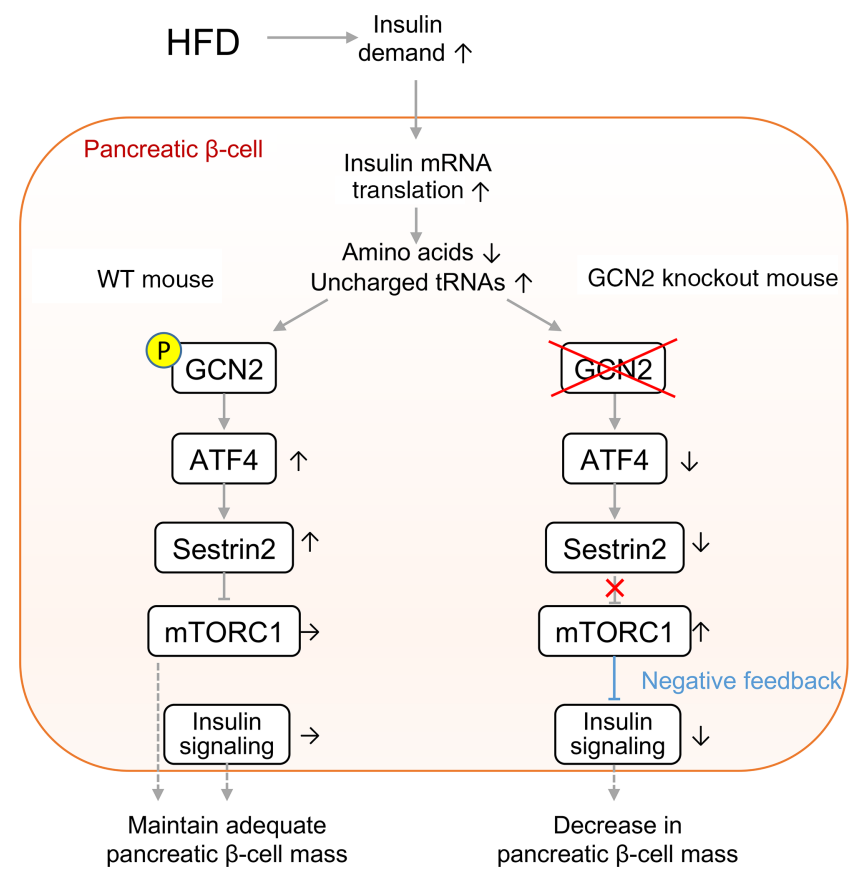

Figure 7. Model for GCN2-dependent regulation of pancreatic $\beta$ cell mass during high-fat feeding.

a decrease in amino acid levels and an increase in uncharged tRNA levels in the islets of WT mice fed a HFD. Insulin is produced predominantly in pancreatic $\beta$ cells, whose volume is $<1 \%$ of the entire pancreas, and proinsulin mRNA constitutes up to $50 \%$ of total mRNA when these cells are stimulated with glucose (52). During high-fat feeding, the chronic need to produce large amounts of insulin likely leads to the consumption of amino acids and the accumulation of uncharged tRNAs. The reduced expression of Slc1a5, which encodes a transporter that mediates the uptake of alanine, serine, cysteine, threonine, glutamate, and asparagine $(53,54)$, may also reflect, in part, the depletion of amino acids in the islets of mice fed a HFD. The attenuation of insulin production has been associated with the expression of tRNA-related genes, including those relevant to tRNA aminoacylation, in pancreatic islets (55). These observations are suggestive of a close relationship between proinsulin mRNA translation and tRNA aminoacylation.

Obesity and insulin resistance result in increased serum levels of amino acids (56), which are associated with an increased risk for the development of T2DM (57-59). We also detected an increase in serum amino acid levels in WT mice fed a HFD, whereas amino acid levels in islets tended to be decreased. GCN2 was first identified as a sensor of amino acid deprivation in Saccharomyces cerevisiae (60). Our results now suggest that a molecule whose original role was to sense amino acid depletion and thereby cope with undernutrition now contributes to the handling of overnutrition associated with the consumption of a HFD in mammals.

On the other hand, oxidative stress has been demonstrated to activate GCN2 $(61,62)$. Because overeating or obesity causes oxidative stress in pancreatic $\beta$ cells, oversuppression of mTORC1 signaling by activated GCN2 possibly leads to pancreatic $\beta$ cell failure, irrespective of the presence or absence of EIF2AK4 SNP rs 2250402 .

The rs2250402 SNP of EIF2AK4 has an overall minor allele frequency of 0.094 , but this frequency is much higher (minor allele frequency 0.267) in the Japanese population (Japanese SNPs, Japan Science and Technology Agency, Tokyo, Japan), and it is also higher in the Chinese population compared with White and African populations (1000 Genomes Project, http://www.1000genomes.org/). Approximately $38 \%$ of our Japanese patient cohort harbored the risk (minor) allele for T2DM. Our finding that subjects with the risk allele exhibited a reduced level of insulin secretion suggests that the function of GCN2 in the pancreatic $\beta$ cells of such individuals might be impaired and that they may be more susceptible to the development of T2DM as a result of pancreatic $\beta$ cell failure in the setting of increased insulin resistance caused by overeating or obesity. Despite the fact that rs 2250402 has a relatively high minor allele frequency in the Japanese population, its association with T2DM did not achieve statistical significance $(P<5.0 \times$ $\left.10^{-7}\right)(4)$. Our findings now suggest that the significance of this association might be increased in individuals 
characterized by overeating or obesity. Based on this hypothesis, early intervention, especially in the form of nutritional guidance, for carriers of the risk allele for EIF2AK4 SNP rs2250402 should be prioritized, because their pancreatic $\beta$ cells would be insufficient for compensatory hyperplasia in response to obesity or overeating. The present results provide an insight into the genetic predisposition to T2DM in nonobese Asian populations, and they may inform a strategy for early intervention, such as nutritional guidance, in those with the risk allele for this SNP.

\section{Methods}

Mice. Eif2ak4 generalized and conditional KO mice (accession nos. CDB0538K and CDB1083K, respectively; http://www2.clst.riken.jp/arg/mutant\%20mice\%20list.html) were generated as described (http:// www2.clst.riken.jp/arg/methods.html). To construct the targeting vectors, genomic fragments of the Eif2ak4 locus were obtained from the bacterial artificial chromosome RP23-450P9 (BACPAC Resources). In the case of systemic $\mathrm{KO}$ mice, a 3.4-kbp region containing exons 11 and 12 of the gene was replaced with a loxP-flanked neomycin resistance (neo) gene cassette (Supplemental Figure 2A). In the case of the $\beta G C N 2^{-/-}$mice, a 590-bp region containing exon 12 of the gene was flanked by loxP sites (Supplemental Figure 2B). Targeted embryonic stem cell clones were microinjected into 8-cell stage embryos of an ICR mouse, frequently used mouse strain for the purpose of prolific, which were then transferred to pseudopregnant ICR females. The resulting chimeras were bred with C57BL/6 mice, and heterozygous offspring were identified by PCR analysis. Primers for genotyping generalized Eif2ak4-null mice were P1 (5'-GTGGGTATGTGCACATGTGGATAC-3') and P2 (5'-CTTCCCTTTCCACTCCCAAAATG-3') for the WT allele (forward, P1; reverse, P2) and P3 (5'-CGCCTTCTTGACGAGTTCTTCTG-3') and P2 for the targeted allele (forward, P1; reverse, P3), which yielded 453-bp and 665-bp products, respectively.

The primers for genotyping the conditional $\mathrm{KO}$ mice were P4 (5'-CATCAGGCGACACTGAAGAAC-3') and P5 (5'-CTGGGTTGCATTTGGCTACTC-3'), which yielded a 333-bp product for the WT allele and a 412-bp product for the floxed allele. The neo cassette was removed by crossing mice heterozygous for the targeted conditional KO allele with Flp-deleter mice. Mice heterozygous for the floxed allele (GCN2 ${ }^{\mathrm{f} / /+}$ mice) were then crossed with mice expressing the Cre recombinase gene under the control of the rat insulin 2 gene promoter (Ins-Cre mice), which were provided by P. Herrera (University of Geneva, Geneva, Switzerland) (63), to generate the KO allele. Mice homozygous for deletion of the GCN2 gene ( $\beta G C N 2^{-/-}$mice) were generated by crossing GCN2 $2^{\mathrm{fl} /+}$ Ins-Cre mice with $\mathrm{GCN} 2^{\mathrm{fl} /+}$ mice. GCN2 $2^{\mathrm{f} / \mathrm{fl}}$ mice were studied as controls.

The mice were maintained on a 12-hour light/12-hour dark cycle. The NCD and HFD were obtained from CLEA Japan and Oriental Bio Service, respectively, and the mice were fed with the respective diet from weaning. Blood glucose and serum insulin concentrations were determined as described previously $(64,65)$. OGTT and ITT were also performed as described previously (34). All experiments were performed with male mice.

Isolation of pancreatic islets. Pancreatic islets were isolated by collagenase digestion (Collagenase P; Roche) and Histopaque (Sigma-Aldrich) density-gradient centrifugation as described previously $(22,66,67)$.

Hepatic triglyceride assay. Portions (50-100 mg) of isolated liver were homogenized and assayed for triglyceride with the use of an enzyme-linked immunosorbent assay kit (Triglyceride E-test; Wako Pure Chemical Industries).

Immunostaining and determination of pancreatic $\beta$ cell mass. To determine pancreatic $\beta$ cell mass, pancreatic tissue was fixed with Bouin's solution, embedded in paraffin, and sectioned at a thickness of $4 \mu \mathrm{m}$. Sectioned pancreatic tissue was stained with guinea pig antibodies to insulin (catalog IR00261, Dako) and rabbit antibodies to glucagon (catalog A0565, Dako), and immune complexes were detected with corresponding secondary antibodies, Cy3-conjugated anti-guinea pig antibody (catalog 706-165-148, Jackson ImmunoResearch Laboratories) and FITC-conjugated anti-rabbit antibody (catalog 711-095-152, Jackson ImmunoResearch Laboratories). Pancreatic $\beta$ cell mass was then quantified as described previously (66). To stain the cell membrane, pancreatic tissue was fixed with paraformaldehyde. Paraffin-depleted sections were subjected to antigen retrieval, and the pancreatic sections were labeled with rabbit antibodies to E-cadherin (catalog 3195S, Cell Signaling Technology) and FITC-conjugated secondary antibodies (catalog 711-095152, Jackson ImmunoResearch Laboratories). TUNEL staining was performed with the use of an Apoptosis in situ Detection Kit (Wako). To detect proliferating cells, pancreatic sections were stained with rabbit antibodies to Ki67 (catalog ab16667, Abcam) and Cy3-conjugated secondary antibodies (catalog 711-165-152, Jackson ImmunoResearch Laboratories). They were stained with insulin to detect pancreatic $\beta$ cells. Nuclei 
were stained with DAPI. Proliferation and apoptosis in pancreatic $\beta$ cells were determined from the number of $\mathrm{Ki}^{+} 7^{+}$and TUNEL ${ }^{+} \beta$ cells divided by the number of islets. Islets $\geq 100 \mu \mathrm{m}$ in diameter were examined.

Cell culture and treatment. INS-1 cells were provided by Kazuya Yamagata (Kumamoto University) and maintained in RPMI 1640 medium (Sigma-Aldrich) supplemented with 10\% FBS. The cells were treated with diazoxide (Sigma-Aldrich), tolbutamide (Sigma-Aldrich), cycloheximide (Sigma-Aldrich), or puromycin (Nacalai Tesque) as indicated.

Transfection with siRNAs. INS-1 cells were transfected with siRNAs targeting GCN2, ATF4, and Sestrin2 (SMARTpool; Dharmacon) as described previously (68).

Adenovirus infection. Mouse Sestrin2 cDNA was cloned with the use of a PrimeScript High Fidelity RT-PCR Kit (Takara Bio Inc.), and an adenovirus encoding Sestrin2 was generated with the use of an Adenovirus Dual Expression Kit (Takara). A control virus encoding $\beta$-galactosidase was described previously (69). INS-1 cells were infected with the control or Sestrin 2 adenoviruses for 24 hours at 48 hours after transfection with GCN2 siRNA.

Immunoblot analysis. Lysates of isolated islets or INS-1 cells were prepared as described previously $(23,59)$. The lysates were probed with antibodies to the following proteins: GCN2 (catalog 3302S), phospho-mTOR (Ser ${ }^{2448}$ ) (catalog 2971S), phospho-S6 kinase (Thr ${ }^{389}$ ) (catalog 9206S), S6 kinase (catalog 2217S), phospho-S6 (Ser ${ }^{235 / 236}$ ) (catalog 2211S), S6 (catalog 2217S), phospho-4EBP1 ( Thr $^{37 / 46}$ ) (catalog 9459S), 4EBP1 (catalog 9452S), phospho-Akt ( $\mathrm{Thr}^{308}$ ) (catalog 9275S), phospho-Akt (Ser ${ }^{473}$ ) (catalog 9271S), Akt (catalog 9272S), phospho-GSK3 $\beta$ (Ser ${ }^{9}$ ) (catalog 9336S), IRS2 (catalog 3089S), phospho-eIF2 $\alpha$ (Ser $^{51}$ ) (catalog 3597S), eIF2 $\alpha$ (catalog 9722S), and TSC2 (catalog 4308S) (all from Cell Signaling Technology); $\beta$-actin (catalog A5316-100UL, Sigma-Aldrich); phospho-GCN2 (catalog ab75836, Abcam); ATF4 (catalog sc-200) and GADD34 (catalog sc-825) (both from Santa Cruz Biotechnology, Dallas, TX); and REDD1 (catalog 10638-1-AP) and Sestrin2 (catalog 10795-1-AP) (both from Proteintech). Band intensity on immunoblots was quantitated with the use of Multi Gauge software (Fujifilm).

Reverse transcription and real-time PCR analysis. Total RNA was isolated from mouse islets or INS-1 cells with the use of an RNeasy Kit (Qiagen). Real-time PCR analysis was performed with the SYBR Green reagent (Promega) and an ABI 7900 sequencer (Invitrogen) as described previously (70). The abundance of target mRNAs was normalized by that of cyclophilin mRNA as the invariant control. Primer sequences for real-time PCR analysis are provided in Supplemental Table 3.

Assay of proinsulin biosynthesis. Proinsulin biosynthesis was assessed as described previously (34). In brief, INS-1 cells or mouse islets were incubated with Click-iT AHA (L-azidohomoalanine) in methionine-free medium for 30 minutes, lysed, and subjected to immunoprecipitation with antibodies to insulin (catalog L6B10, Cell Signaling Technology; catalog ab7842, Abcam). The immunoprecipitates were reacted with biotin-alkyne (Invitrogen), subjected to polyacrylamide gel electrophoresis, and probed with horseradish peroxidase-conjugated streptavidin (Cell Signaling Technology).

Amino acid measurement. Methanol $(500 \mu \mathrm{L})$ containing $50 \mu \mathrm{M}$ of each internal standard was added to $30 \mathrm{mg}$ liver or hypothalamic tissue or 300 pancreatic islets, and the mixtures were homogenized using a bead crusher ( $\mu \mathrm{T}$-12; Taitec Corp.). The homogenates were mixed with $200 \mu \mathrm{L}$ distilled water and $500 \mu \mathrm{L}$ $\mathrm{CHCl}_{3}$. The mixture was then centrifuged at $2300 \mathrm{~g}$ for 5 minutes at $4^{\circ} \mathrm{C}$, and the resultant supernatants containing water-soluble metabolites were depleted of protein by filtration ( 5 -kDa filter; Merck KGaA) and freeze-dried under a vacuum.

As for serum, $50 \mu \mathrm{L}$ serum was mixed with a solvent solution $(450 \mu \mathrm{L}$ methanol, $200 \mu \mathrm{L}$ distilled water, $500 \mu \mathrm{L} \mathrm{CHCl}_{3}$ ) containing $50 \mu \mathrm{M}$ of each internal standard. The mixture was then centrifuged at $2300 \mathrm{~g}$ for 5 minutes at $4^{\circ} \mathrm{C}$. The resultant supernatant $(450 \mu \mathrm{L})$ containing water-soluble metabolites was depleted of protein by filtration and freeze-dried under a vacuum.

The freeze-dried samples were dissolved in ultrapure water and analyzed by capillary electrophoresis-TOF MS (7100CE and 6200TOF LC/MS; Agilent Technologies). Metabolite concentrations were calculated on the basis of the simultaneous analysis of the standards.

Aminoacyl-tRNA measurement. Islets were disrupted in Acid-Phenol:Chloroform, pH 4.5 (Invitrogen), and centrifuged at $12,000 \mathrm{~g}$ for 15 minutes at $4^{\circ} \mathrm{C}$. The supernatant was mixed with isopropanol, and total RNA was precipitated by centrifugation at $12,000 \mathrm{~g}$ for 15 minutes at $4^{\circ} \mathrm{C}$. Total RNA was washed once with $0.3 \mathrm{M}$ sodium acetate $(\mathrm{pH} 4.5)$ in $70 \%$ ethanol and suspended in $10 \mathrm{mM}$ sodium acetate ( $\mathrm{pH} 4.5)$. RNA was adjusted to a concentration of $1 \mathrm{mg} / \mathrm{mL}$. Deacylation was performed by the addition of an equal volume of $100 \mathrm{mM}$ Tris- $\mathrm{HCl}(\mathrm{pH} 9.0)$, followed by incubation at $75^{\circ} \mathrm{C}$ for 15 minutes. Subsequently, the 
$\mathrm{pH}$ of the samples was adjusted to about 6 by the addition of glacial acetic acid, and the concentrations of free amino acids were measured using liquid chromatography-MS (LC-MS) (Agilent 6460). The mobile phase was as follows: A, $0.2 \%$ formic acid and $0.2 \%$ acetic acid; B, $100 \mathrm{mM}$ ammonium acetate in water/ methanol (50:50). Amino acids were measured using a multiple reaction monitoring method.

Northern blot analysis. Total RNA was purified and suspended in an acidic solvent. Aminoacylated tRNA was detected as described previously (71). Briefly, tRNA was separated by acid-urea polyacrylamide gel electrophoresis. tRNA was then transferred to a nylon membrane (Hybond-N+; GE Healthcare), followed by UV cross-linking. The membrane was hybridized with a ${ }^{32} \mathrm{P}$-labeled probe to detect tRNA-Ser. After extensive washing, the membrane was exposed to a phosphorimager screen and visualized (FLA 7000; GE Healthcare).

Human subjects and analysis. A total of 111 individuals, consisting of 33 with NGT, 15 with IGT, and 63 with T2DM, were included in this study. SNPs were genotyped for association analyses with the use of a TaqMan assay on an ABI 7900 sequencer (Invitrogen). In 1 of the 11 subjects carrying the risk allele ( $A C$ and $C C$ genotypes) in the NGT group and in 1 of the 6 subjects without the risk allele ( $A A$ genotype) in the IGT group, the hyperinsulinemic-euglycemic clamp test was not conducted. In 5 of the 40 subjects without the risk allele ( $A A$ genotype) and in 4 of the 23 subjects with the risk allele ( $A C$ and $C C$ genotypes) in the T2DM group, the 75-g OGTT was not conducted. The 75-g OGTT and consecutive hyperglycemic and hyperinsulinemic-euglycemic clamp test were performed as described previously (72). For the 75-g OGTT, Trelan-G75 (Shimizu Shiyaku) was administered orally to all subjects after an overnight fast. Plasma glucose and immunoreactive insulin (IRI) were measured before the administration of Trelan-G75 and at 0 , 30,60 , and 120 minutes thereafter. We calculated the insulinogenic index, which was defined as the ratio of the increase of insulin to plasma glucose at 30 minutes after glucose loading ( $\Delta$ insulin $0-30 \mathrm{~min} / \Delta$ plasma glucose 0-30 $\mathrm{min}$ ). For the clamp test, for the first 90 minutes, a hyperglycemic clamp was performed by i.v. infusion of a bolus of glucose $\left(9622 \mathrm{mg} / \mathrm{m}^{2}\right)$ over 15 minutes, followed by a variable amount of glucose to maintain the plasma glucose level at $200 \mathrm{mg} / \mathrm{dL}$. At 10 minutes after the end of the hyperglycemic clamp, a 120-minute hyperinsulinemic-euglycemic clamp was initiated by i.v. infusion of normal human insulin (Lilly) at a rate of $40 \mathrm{mU} / \mathrm{m}^{2} / \mathrm{min}$ and with a target plasma glucose level of $90 \mathrm{mg} / \mathrm{dL}$. For the NGT and IGT subjects whose fasting plasma glucose levels were $<90 \mathrm{mg} / \mathrm{dL}$, the plasma glucose concentration was clamped at the fasting level. First-phase insulin secretion during the hyperglycemic clamp was defined as the incremental area under the serum IRI concentration curve $\left(\mu \mathrm{U} / \mathrm{mL} / \mathrm{min}\right.$ ) from $0-10$ minutes $\left(\mathrm{AUC}_{\mathrm{I}}\right.$ ${ }_{\mathrm{RI}}$ ). The insulin sensitivity index was calculated by dividing the mean glucose infusion rate during the final 30 minutes of the clamp $(\mathrm{mg} / \mathrm{kg} / \mathrm{min})$ by both the plasma glucose $(\mathrm{mg} / \mathrm{dL})$ and serum insulin $(\mu \mathrm{U} / \mathrm{mL})$ levels at the end of the clamp and then multiplying the resulting value by 100 . A clamp-based analog of the disposition index was calculated as the product of $\mathrm{AUC}_{\mathrm{IRI} 10}$ and the insulin sensitivity index. Analyses were carried out using the statistical package JMP version 8.0. These variables and logarithmic-converted variables were normally distributed according to the Shapiro-Wilk test. The correlations between BMI and the disposition index were analyzed by Pearson's correlation analysis.

Statistics. Quantitative data are presented as the mean \pm SEM, and differences between means were assessed with Student's $t$ test (2-tailed). $P<0.05$ was considered statistically significant.

Study approval. The animal studies were approved by the Animal Ethics Committee of Kobe University Graduate School of Medicine and the IACUC of RIKEN Kobe Branch. The human studies were approved by the Ethics Committee of Kobe University Hospital, and written informed consent was obtained from all subjects prior to inclusion in the study. Participants were identified by number, not by name.

\section{Author contributions}

AK, AF, KM, RY, ES, TT, NY, YI, KI, TA, and FYW performed the experiments and acquired the data. AK, SIA, MK, and YK conceived and designed the experiments. AK, SIA, MKK, TM, YH, NY, HI, KT, and YK analyzed and interpreted the data. AK, SIA, AB, MM, WO, SS, MK, and YK contributed to the discussion and edited the manuscript. AK, SIA, and YK wrote the manuscript. All authors critically reviewed the manuscript for intellectual content and approved the final version.

\section{Acknowledgments}

We thank M. Nagano, M. Oya, A. Tanida, and S. Hirahara for technical assistance. This research was supported by a grant for the Cooperative Network of Unique Science and Technology for Economic Revitalization from the Ministry of Education, Culture, Sports, Science and Technology (MEXT) of Japan 
(to MK); a grant-in-aid for creative scientific research from MEXT (18GS0317 to MK); grants-in-aid for creative scientific research from MEXT (22590981, 25461352, and 17H01966 to YK); grants-in-aid for creative scientific research from MEXT (26461382 and 17K09882 to SIA); grants-in-aid for creative scientific research from MEXT (24790944, 26750040, and 16K09803 to MKK); a grant-in-aid for creative scientific research from MEXT (16K16277 to AK); grants from Hyogo Science and Technology Association (to YK); grants from The Naito Foundation (to YK); grants from The Food Science Institute Foundation (to SIA); and grants from Mishima Kaiun Memorial Foundation (to AK).

Address correspondence to: Shun-ichiro Asahara or Yoshiaki Kido, Division of Diabetes and Endocrinology, Department of Internal Medicine, Kobe University Graduate School of Medicine, 7-5-1, Kusunoki-cho, Chuo-ku, Kobe 650-0017, Japan. Phone: 81.78.796.4549; E-mail: asahara@med.kobe-u.ac.jp (SI Aashara); kido@med.kobe-u.ac.jp (Y Kido).

1. Yoon KH, et al. Epidemic obesity and type 2 diabetes in Asia. Lancet. 2006;368(9548):1681-1688

2. Ma RC, Chan JC. Type 2 diabetes in East Asians: similarities and differences with populations in Europe and the United States. Ann N Y Acad Sci. 2013;1281:64-91.

3. Saisho Y, Butler AE, Manesso E, Elashoff D, Rizza RA, Butler PC. $\beta$-cell mass and turnover in humans: effects of obesity and aging. Diabetes Care. 2013;36(1):111-117.

4. Kou K, Saisho Y, Satoh S, Yamada T, Itoh H. Change in $\beta$-cell mass in Japanese nondiabetic obese individuals. J Clin Endocrinol Metab. 2013;98(9):3724-3730.

5. Yasuda K, et al. Variants in KCNQ1 are associated with susceptibility to type 2 diabetes mellitus. Nat Genet. 2008;40(9):1092-1097.

6. Miyake K, et al. Construction of a prediction model for type 2 diabetes mellitus in the Japanese population based on 11 genes with strong evidence of the association. J Hum Genet. 2009;54(4):236-241.

7. Dong J, Qiu H, Garcia-Barrio M, Anderson J, Hinnebusch AG. Uncharged tRNA activates GCN2 by displacing the protein kinase moiety from a bipartite tRNA-binding domain. Mol Cell. 2000;6(2):269-279.

8. Zaborske JM, et al. Genome-wide analysis of tRNA charging and activation of the eIF2 kinase Gcn2p. J Biol Chem. 2009;284(37):25254-25267.

9. Sood R, Porter AC, Olsen DA, Cavener DR, Wek RC. A mammalian homologue of GCN2 protein kinase important for translational control by phosphorylation of eukaryotic initiation factor-2alpha. Genetics. 2000;154(2):787-801.

10. Lu PD, Harding HP, Ron D. Translation reinitiation at alternative open reading frames regulates gene expression in an integrated stress response. J Cell Biol. 2004;167(1):27-33.

11. Kilberg MS, Shan J, Su N. ATF4-dependent transcription mediates signaling of amino acid limitation. Trends Endocrinol Metab. 2009;20(9):436-443.

12. Hao S, et al. Uncharged tRNA and sensing of amino acid deficiency in mammalian piriform cortex. Science. 2005;307(5716):1776-1778.

13. Maurin AC, et al. The GCN2 kinase biases feeding behavior to maintain amino acid homeostasis in omnivores. Cell Metab. 2005;1(4):273-277.

14. Costa-Mattioli M, et al. Translational control of hippocampal synaptic plasticity and memory by the eIF2alpha kinase GCN2 Nature. 2005;436(7054):1166-1173.

15. Munn DH, et al. GCN2 kinase in T cells mediates proliferative arrest and anergy induction in response to indoleamine 2,3-dioxygenase. Immunity. 2005;22(5):633-642.

16. Ravindran R, et al. The amino acid sensor GCN2 controls gut inflammation by inhibiting inflammasome activation. Nature. 2016;531(7595):523-527.

17. Guo F, Cavener DR. The GCN2 eIF2alpha kinase regulates fatty-acid homeostasis in the liver during deprivation of an essential amino acid. Cell Metab. 2007;5(2):103-114.

18. Xu X, Hu J, McGrath BC, Cavener DR. GCN2 regulates the CCAAT enhancer binding protein beta and hepatic gluconeogenesis. Am J Physiol Endocrinol Metab. 2013;305(8):E1007-E1017.

19. Xiao F, et al. Leucine deprivation increases hepatic insulin sensitivity via GCN2/mTOR/S6K1 and AMPK pathways. Diabetes. 2011;60(3):746-756.

20. Wagner H, Alvarsson M, Mannheimer B, Degerblad M, Östenson CG. No Effect of High-Dose Vitamin D Treatment on $\beta$-Cell Function, Insulin Sensitivity, or Glucose Homeostasis in Subjects With Abnormal Glucose Tolerance: A Randomized Clinical Trial. Diabetes Care. 2016;39(3):345-352.

21. Michaliszyn SF, et al. Metabolomic profiling of amino acids and $\beta$-cell function relative to insulin sensitivity in youth. $J$ Clin Endocrinol Metab. 2012;97(11):E2119-E2124.

22. Shigeyama Y, et al. Biphasic response of pancreatic beta-cell mass to ablation of tuberous sclerosis complex 2 in mice. Mol Cell Biol. 2008;28(9):2971-2979.

23. Bartolomé A, et al. Pancreatic $\beta$-cell failure mediated by mTORC1 hyperactivity and autophagic impairment. Diabetes. 2014;63(9):2996-3008.

24. Dever TE, Feng L, Wek RC, Cigan AM, Donahue TF, Hinnebusch AG. Phosphorylation of initiation factor 2 alpha by protein kinase GCN2 mediates gene-specific translational control of GCN4 in yeast. Cell. 1992;68(3):585-596.

25. Harding HP, et al. Regulated translation initiation controls stress-induced gene expression in mammalian cells. Mol Cell. 2000;6(5):1099-1108. 
26. DeYoung MP, Horak P, Sofer A, Sgroi D, Ellisen LW. Hypoxia regulates TSC1/2-mTOR signaling and tumor suppression through REDD1-mediated 14-3-3 shuttling. Genes Dev. 2008;22(2):239-251.

27. Watanabe R, et al. GADD34 inhibits mammalian target of rapamycin signaling via tuberous sclerosis complex and controls cell survival under bioenergetic stress. Int J Mol Med. 2007;19(3):475-483.

28. Chantranupong L, et al. The Sestrins interact with GATOR2 to negatively regulate the amino-acid-sensing pathway upstream of mTORC1. Cell Rep. 2014;9(1):1-8

29. Peng M, Yin N, Li MO. Sestrins function as guanine nucleotide dissociation inhibitors for Rag GTPases to control mTORC1 signaling. Cell. 2014;159(1):122-133.

30. Parmigiani A, et al. Sestrins inhibit mTORC1 kinase activation through the GATOR complex. Cell Rep. 2014;9(4):1281-1291.

31. Kim JS, et al. Sestrin2 inhibits mTORC1 through modulation of GATOR complexes. Sci Rep. 2015;5:9502.

32. Itoh N, Okamoto H. Translational control of proinsulin synthesis by glucose. Nature. 1980;283(5742):100-102.

33. Wicksteed B, Alarcon C, Briaud I, Lingohr MK, Rhodes CJ. Glucose-induced translational control of proinsulin biosynthesis is proportional to preproinsulin mRNA levels in islet beta-cells but not regulated via a positive feedback of secreted insulin. $J$ Biol Chem. 2003;278(43):42080-42090

34. Kanno A, et al. Compensatory hyperinsulinemia in high-fat diet-induced obese mice is associated with enhanced insulin translation in islets. Biochem Biophys Res Commun. 2015;458(3):681-686.

35. Hashimoto N, et al. Ablation of PDK1 in pancreatic beta cells induces diabetes as a result of loss of beta cell mass. Nat Genet. 2006;38(5):589-593.

36. Blandino-Rosano M, et al. Loss of mTORC1 signalling impairs $\beta$-cell homeostasis and insulin processing. Nat Commun. 2017;8:16014.

37. Fraenkel M, et al. mTOR inhibition by rapamycin prevents beta-cell adaptation to hyperglycemia and exacerbates the metabolic state in type 2 diabetes. Diabetes. 2008;57(4):945-957.

38. Barlow AD, Nicholson ML, Herbert TP. Evidence for rapamycin toxicity in pancreatic $\beta$-cells and a review of the underlying molecular mechanisms. Diabetes. 2013;62(8):2674-2682.

39. Fang Y, et al. Duration of rapamycin treatment has differential effects on metabolism in mice. Cell Metab. 2013;17(3):456-462.

40. Nie J, et al. SAD-A kinase controls islet $\beta$-cell size and function as a mediator of mTORC1 signaling. Proc Natl Acad Sci USA. 2013;110(34):13857-13862.

41. Kitamura T, et al. The forkhead transcription factor Foxo1 links insulin signaling to Pdx1 regulation of pancreatic beta cell growth. J Clin Invest. 2002;110(12):1839-1847.

42. Inoki $\mathrm{K}, \mathrm{Li}$ Y, Xu T, Guan KL. Rheb GTPase is a direct target of TSC2 GAP activity and regulates mTOR signaling. Genes Dev. 2003;17(15):1829-1834.

43. Bar-Peled L, et al. A Tumor suppressor complex with GAP activity for the Rag GTPases that signal amino acid sufficiency to mTORC1. Science. 2013;340(6136):1100-1106.

44. Kim E, Goraksha-Hicks P, Li L, Neufeld TP, Guan KL. Regulation of TORC1 by Rag GTPases in nutrient response. Nat Cell Biol. 2008;10(8):935-945.

45. Sancak Y, et al. The Rag GTPases bind raptor and mediate amino acid signaling to mTORC1. Science. 2008;320(5882):1496-1501

46. Um SH, et al. Absence of S6K1 protects against age- and diet-induced obesity while enhancing insulin sensitivity. Nature. 2004;431(7005):200-205.

47. Hatanaka M, et al. Palmitate induces mRNA translation and increases ER protein load in islet $\beta$-cells via activation of the mammalian target of rapamycin pathway. Diabetes. 2014;63(10):3404-3415.

48. Yuan T, et al. Reciprocal regulation of mTOR complexes in pancreatic islets from humans with type 2 diabetes. Diabetologia. 2017;60(4):668-678

49. Ravishankar B, et al. The amino acid sensor GCN2 inhibits inflammatory responses to apoptotic cells promoting tolerance and suppressing systemic autoimmunity. Proc Natl Acad Sci USA. 2015;112(34):10774-10779.

50. Berlanga JJ, Santoyo J, De Haro C. Characterization of a mammalian homolog of the GCN2 eukaryotic initiation factor 2alpha kinase. Eur J Biochem. 1999;265(2):754-762.

51. Zhang P, et al. The GCN2 eIF2alpha kinase is required for adaptation to amino acid deprivation in mice. Mol Cell Biol. 2002;22(19):6681-6688.

52. Schuit FC, In't Veld PA, Pipeleers DG. Glucose stimulates proinsulin biosynthesis by a dose-dependent recruitment of pancreatic beta cells. Proc Natl Acad Sci USA. 1988;85(11):3865-3869.

53. Scalise M, Pochini L, Panni S, Pingitore P, Hedfalk K, Indiveri C. Transport mechanism and regulatory properties of the human amino acid transporter ASCT2 (SLC1A5). Amino Acids. 2014;46(11):2463-2475

54. Kanai Y, et al. The SLC1 high-affinity glutamate and neutral amino acid transporter family. Mol Aspects Med. 2013;34(2-3):108-120

55. Szabat M, et al. Reduced Insulin Production Relieves Endoplasmic Reticulum Stress and Induces $\beta$ Cell Proliferation. Cell Metab. 2016;23(1):179-193.

56. Tai ES, et al. Insulin resistance is associated with a metabolic profile of altered protein metabolism in Chinese and Asian-Indian men. Diabetologia. 2010;53(4):757-767.

57. Wang TJ, et al. Metabolite profiles and the risk of developing diabetes. Nat Med. 2011;17(4):448-453.

58. Cheng S, et al. Metabolite profiling identifies pathways associated with metabolic risk in humans. Circulation. 2012;125(18):2222-2231.

59. Floegel A, et al. Identification of serum metabolites associated with risk of type 2 diabetes using a targeted metabolomic approach. Diabetes. 2013;62(2):639-648.

60. Roussou I, Thireos G, Hauge BM. Transcriptional-translational regulatory circuit in Saccharomyces cerevisiae which involves the GCN4 transcriptional activator and the GCN2 protein kinase. Mol Cell Biol. 1988;8(5):2132-2139.

61. Krohn M, Skjølberg HC, Soltani H, Grallert B, Boye E. The G1-S checkpoint in fission yeast is not a general DNA damage checkpoint. J Cell Sci. 2008;121(Pt 24):4047-4054

62. Anda S, Zach R, Grallert B. Activation of Gcn2 in response to different stresses. PLoS One. 2017;12(8):e0182143.

63. Herrera PL. Adult insulin- and glucagon-producing cells differentiate from two independent cell lineages. Development. 
2000;127(11):2317-2322.

64. Kido Y, et al. Tissue-specific insulin resistance in mice with mutations in the insulin receptor, IRS-1, and IRS-2. J Clin Invest. 2000;105(2):199-205.

65. Hashimoto N, et al. PKClambda regulates glucose-induced insulin secretion through modulation of gene expression in pancreatic beta cells. J Clin Invest. 2005;115(1):138-145.

66. Matsuda T, et al. Ablation of C/EBPbeta alleviates ER stress and pancreatic beta cell failure through the GRP78 chaperone in mice. J Clin Invest. 2010;120(1):115-126.

67. Koyanagi M, et al. Ablation of TSC2 enhances insulin secretion by increasing the number of mitochondria through activation of mTORC1. PLoS One. 2011;6(8):e23238.

68. Asahara S, et al. Ras-related C3 botulinum toxin substrate 1 (RAC1) regulates glucose-stimulated insulin secretion via modulation of F-actin. Diabetologia. 2013;56(5):1088-1097.

69. Inoue H, et al. Role of STAT-3 in regulation of hepatic gluconeogenic genes and carbohydrate metabolism in vivo. Nat Med. 2004;10(2):168-174.

70. Asahara S, et al. Paternal allelic mutation at the Kcnq1 locus reduces pancreatic $\beta$-cell mass by epigenetic modification of Cdkn1c. Proc Natl Acad Sci USA. 2015;112(27):8332-8337.

71. Janssen BD, Diner EJ, Hayes CS. Analysis of aminoacyl- and peptidyl-tRNAs by gel electrophoresis. Methods Mol Biol. 2012;905:291-309.

72. Ohashi K, et al. Glucose Homeostatic Law: Insulin Clearance Predicts the Progression of Glucose Intolerance in Humans. PLoS One. 2015;10(12):e0143880. 